\title{
Fractional Model and Numerical Algorithms for Predicting COVID-19 with Isolation and Quarantine Strategies
}

\author{
Abdelouahed Alla Hamou ${ }^{1}$ (D) - Elhoussine Azroul ${ }^{1}$ - Abdelilah Lamrani Alaoui ${ }^{1,2}$
}

Accepted: 6 May 2021 / Published online: 30 June 2021

(c) The Author(s), under exclusive licence to Springer Nature India Private Limited 2021

\begin{abstract}
In December 2019, a new outbreak in Wuhan, China has attracted world-wide attention, the virus then spread rapidly in most countries of the world, the objective of this paper is to investigate the mathematical modelling and dynamics of a novel coronavirus (COVID-19) with Caputo-Fabrizio fractional derivative in the presence of quarantine and isolation strategies. The existence and uniqueness of the solutions for the fractional model is proved using fixed point iterations, the fractional model are shown to have disease-free and an endemic equilibrium point. We construct a fractional version of the four-steps Adams-Bashforth method as well as the error estimate of this method. We have used this method to determine the numerical scheme of this model and Matlab program to illustrate the evolution of the virus in some countries (Morocco, Qatar, Brazil and Mexico) as well as to support theoretical results. The Least squares fitting is a way to find the best fit curve or line for a set of points, so we apply this method in this paper to construct an algorithm to estimate the parameters of fractional model as well as the fractional order, this model gives an estimate better than that of classical model.
\end{abstract}

Keywords Caputo-Fabrizio derivative · COVID-19 · SEIR epidemic model · Fractional differential equations - Fractional Adams-Bashforth method

Mathematics Subject Classification 65D05 $\cdot$ 65R20 $\cdot$ 26A33 $\cdot$ 93E24

\section{Introduction}

In December 2019, an unidentified virus was found in Wuhan, Hubei province, China. The responsible virus was later confirmed as a new coronavirus [23]. The World Health Organization (WHO) temporarily named the virus as the novel coronavirus 2019 (2019-nCoV), and the disease as coronavirus disease 2019 (COVID-19) on 11 February 2020. The first

$凶$ Abdelouahed Alla Hamou

abdelouahed.allahamou@gmail.com

1 Laboratory of Mathematical Analysis and Applications, Faculty of Sciences Dhar Al Mahraz, Sidi Mohamed Ben Abdellah University, B.P. 1796, 30000 Fez, Morocco

2 Department of Mathematics, Regional Center of Education and Professional Training, B.P. 49, 30000 Fez, Morocco 
confirmed case of the virus was discovered on 17 November 2019 in Hubei. As of 5 July 2020, more than 11.1 million cases have been reported in 188 countries in the world, which resulted in more than 528,000 deaths. Over 6.03 million people recovered [57].

The progress of fractional calculus in the last few years has been very rapid due to its applicability in many fields, such as physical problems [15,23,51,58], engineering mechanics [9], epidemiological models [6,14,17,18,26,29,44,45,47], image processing [28,59], chaos theory $[10,52]$ and others. There are in the literature several definitions of the fractional derivative, the most famous of these is the definition of Caputo, Riemann-Liouville, Grünwald-Letnikov and Hadamard derivative, the new operators with non-singular kernels appear in this domain of mathematics, namely the Caputo-Fabrizio derivative [19] and the Atangana-Baleanu-Caputo derivative [8].

A number of epidemiological models with fractional derivative have been developed to understand the transmission dynamics of COVID-19 and other infectious diseases outbreak from various aspects. We mention them, Kumar et al. [33] have been studied fractional SIRS-SI model describing the transmission of malaria disease by using the Caputo-Fabrizio fractional operator. Tuan et al. [53] studied a mathematical model for COVID-19 transmission by using the Caputo fractional derivative. Singh et al. [49] studied the numerical solution of SEIAR model with Grünwald-Leitnikov derivative. Abdo et al. [1] have investigated the mathematical model of novel coronavirus (COVID-19) depending of fourteen nonlinear FDEs with Atangana-Baleanu-Caputo fractional derivative. Peter et al. [42] investigated a fractional order mathematical model of COVID-19 in Nigeria using Atangana-Aaleanu derivative. Ahmad et al. [2] Studied the fractional model and dynamics of COVID-19 in Pakistan using Atangana Baleanau operators. Nisar et al. [41] Proposed a SIRD model of COVID-19 with Caputo fractional derivative. Rezapour et al. [44] presented a new mathematical model for the transmission of Zika virus between humans as well as between humans and mosquitoes using Caputo derivative. Baleanu et al. [16] proposed a new fractional model for human liver involving Caputo- Fabrizio derivative. Rezapour et al. [43] studied the fractionalorder model for the anthrax disease between animals based on the Caputo-Fabrizio derivative. Dokuyucu and Dutta [26] examined the Ebola virus model using the fractional derivative and the integral operator proposed by Caputo and Fabrizio. Area et al. [6] have been studied the classical and Caputo fractional order SEIR (susceptible, exposed, infections, removed) Ebola epidemic model and its comparison with real data. Tulu et al. [54] developed the Caputo fractional mathematical model of the Ebola virus with a quarantine strategy and the presence of the vaccine, using both the Euler method and one of the top ten most influential algorithms known as Markov Chain Monte Carlo (MCMC) method. Singh et al. [48] have been studied the fractional epidemiological model for computer viruses using Caputo-Fabrizio fractional derivative. Singh et al. [47] studied the diabetes model and its complications with the CF-fractional derivative. Mohammadi et al. [38] used Caputo-Fabrizio fractional derivative to model hearing loss in children caused by the mumps virus. Higazy [30] suggested a SIDARTHE model for COVID-19 pandemic by using Caputo fractional derivative. Zhang et al. [63] applied the fractional SEIRD Model to the real data of the COVID-19 for China using Caputo fractional derivative. Some other outstanding studies of COVID-19 by fractional derivative have been made in $[4,5,7,24,31,34,37,46,60,62]$.

Always when a new virus appears, researchers look for effective ways to control the virus, including vaccination, isolation, and quarantine. In the absence of the vaccine, the isolation and quarantine strategies remain effective to mitigate and eliminate the impact of the virus (see $[12,20-22,27,64])$. In this current study, we modify the general SEIR epidemiological model for the effects of isolation and quarantine strategies on COVID-19 transmission, to become a fractional order model of type Caputo-Fabrezio fractional derivative. The main objective 
in this work is to provide a new discussion and new tools for developing epidemiological models with Caputo-Fabrizio derivative, this work is motivated by all these results that prove the efficiency of fractional derivative, to our best knowledge, such problem has never been studied previously for the fractional case. We will start this article in the theoretical part by proving the existence and uniqueness of the solutions, then we will determine the equilibrium and the basic reproduction number of the model. We construct a fractional version of the four-steps Adams-Bashforth method as well as the error estimate of this method.

In machine learning, the Least squares fitting is a way to find the best fit curve or line for a set of points, so we apply this method in this paper to construct an algorithm to estimate the parameters of fractional model as well as the fractional order, this model gives an estimate better than that of classical model. The paper is organized as follows. In Section 2, we present the basic theory of the Caputo-Fabrizio derivative. The clasical and fractional model are formulated in Section 3. The basic roprodoction number and the disease-free equilibrium are given in Section 4. The fixed point iterations is applied to prove existence and uniqueness results in Section 5. Using fractional m-step Adams-Bashforth scheme with CF derivative, the numerical solution of the proposed model is obtained in Section 6. Simulation results are presented in Section 7. Finally, the present work is concluded in Section 8.

\section{Preliminaries}

Recalled here some background material for the Caputo-Fabrizio fractional derivatives, see $[3,19,32]$ for details.

Definition 1 ([19]) Let $u \in H^{1}(a, b), b>a, 0<\alpha<1$, the time fractional Caputo-Fabrizio fractional differential operator is defined by

$$
{ }^{C F} D_{t}^{\alpha} u(t)=\frac{M(\alpha)}{(1-\alpha)} \int_{\mathrm{a}}^{t} \exp \left[-\frac{\alpha(t-x)}{1-\alpha}\right] u^{\prime}(x) d x, \quad t \geq 0 .
$$

where $M$ is a normalization function which depends on $\alpha$ and gives $M(0)=M(1)=1$, where ${ }^{C F} D_{t}^{\alpha} u(t)=0$, if $u$ is a constant function. The definition is also written if the function does not belong to $\mathrm{H}^{1}(a, b)$

$$
{ }^{C F} D_{\mathrm{t}}^{\alpha}(u(t))=\frac{\alpha M(\alpha)}{1-\alpha} \int_{\mathrm{a}}^{\mathrm{t}}(u(t)-u(x)) \exp \left[-\frac{\alpha(t-x)}{1-\alpha}\right] d x .
$$

The corresponding integral was described by Jorge and Juan [32].

Definition 2 ([32]) Let $0<\alpha<1$, then the CF fractional integral operator of order $\alpha$ given by

$$
C F I_{t}^{\alpha} u(t)=\frac{2(1-\alpha)}{(2-\alpha) M(\alpha)} u(t)+\frac{2 \alpha}{(2-\alpha) M(\alpha)} \int_{0}^{t} u(s) d s, \quad t \geq 0 .
$$

\section{Model Formulation}

In more detail, we have studied a fractional SEIR epidemiological model with quarantine and isolation strategies. The total population is divided into seven groups, namely susceptible $(S)$, exposed $(E)$, infectious but not yet symptomatic, infectious with symptoms $(I)$ and recovered $(R)$ compartments, as well as quarantined susceptible $\left(S_{q}\right)$, quarantined exposed $\left(E_{q}\right)$ and 


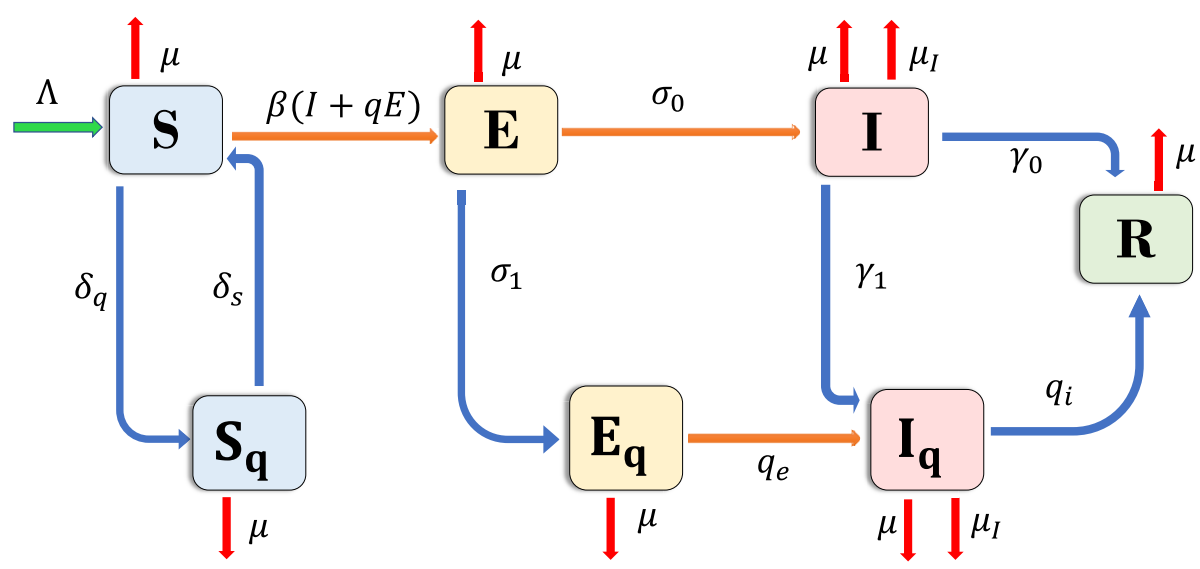

Fig. 1 Schematic diagram of the model compartments and parameters

isolated infected $\left(I_{q}\right)$ compartments. Where susceptible individuals can be quarantined at a rate of $\delta_{s}$ and then returned to the pool of susceptible individuals once it is determined they are uninfected at a rate of $\delta_{q}$. The unquarantined susceptible individuals, if infected, move to the compartment $E$ at a rate of $\beta(I+q E)$ where $\beta$ is the transmission incidence rate and $q$ is the fraction of transmission rate for exposed, also the exposed individuals develop symptoms at a rate $\sigma_{0}$ and are assumed to be quarantined at a rate $\sigma_{1}$, not only-but also the exposed quarantined individuals can be isolated at rate $q_{e}$ they also recover after isolation at rate $q_{i}$. Likewise the infected symptomatically individuals develop symptoms and can be isolated at rate $\gamma_{1}$, in addition they recover from the disease at rates $\gamma_{0}$, finally $\Lambda$ is the recruitment rate, $\mu$ represent natural death rate and $\mu_{I}$ show the death rate of infected human individuals with the coronavirus disease 2019.

The transfer diagram for this model is described by Fig. 1 and the classical version of this model formulated by the following system of ODEs:

$$
\left\{\begin{array}{l}
\frac{\mathrm{d} S}{\mathrm{~d} t}=\Lambda-\beta S(I+q E)+\delta_{q} S_{q}-\left(\delta_{S}+\mu\right) S, \\
\frac{\mathrm{d} E}{\mathrm{~d} t}=\beta S(I+q E)-\left(\sigma_{0}+\sigma_{1}+\mu\right) E, \\
\frac{\mathrm{d} I}{\mathrm{~d} t}=\sigma_{0} E-\left(\gamma_{0}+\gamma_{1}+\mu+\mu_{I}\right) I, \\
\frac{\mathrm{d} R}{\mathrm{~d} t}=\gamma_{0} I+q_{i} I_{q}-\mu R, \\
\frac{\mathrm{d} S_{q}}{\mathrm{~d} t}=\delta_{S} S-\left(\delta_{q}+\mu\right) S_{q}, \\
\frac{\mathrm{d} E_{q}}{\mathrm{~d} t}=\sigma_{1} E-\left(q_{e}+\mu\right) E_{q}, \\
\frac{\mathrm{d} I_{q}}{\mathrm{~d} t}=q_{e} E_{q}+\gamma_{1} I-\left(q_{i}+\mu+\mu_{I}\right) I_{q},
\end{array}\right.
$$

\section{Fractional Model}

Now replacing the classical derivative in (1) by Caputo-Fabrizio derivative we obtain by moment closure, the following system of fractional ODEs : 


$$
\left\{\begin{array}{l}
D_{t}^{\alpha} S=\Lambda-\beta S(I+q E)+\delta_{q} S_{q}-\left(\delta_{s}+\mu\right) S, \\
D_{t}^{\alpha} E=\beta S(I+q E)-\left(\sigma_{0}+\sigma_{1}+\mu\right) E \\
D_{t}^{\alpha} I=\sigma_{0} E-\left(\gamma_{0}+\gamma_{1}+\mu+\mu_{I}\right) I \\
D_{t}^{\alpha} R=\gamma_{0} I+q_{i} I_{q}-\mu R \\
D_{t}^{\alpha} S_{q}=\delta_{s} S-\left(\delta_{q}+\mu\right) S_{q} \\
D_{t}^{\alpha} E_{q}=\sigma_{1} E-\left(q_{e}+\mu\right) E_{q} \\
D_{t}^{\alpha} I_{q}=q_{e} E_{q}+\gamma_{1} I-\left(q_{i}+\mu+\mu_{I}\right) I_{q}
\end{array}\right.
$$

with the initial conditions $S(0)=S_{0} \geq 0, E(0)=E_{0} \geq 0, I(0)=I_{0} \geq 0 ; R(0)=R_{0} \geq 0$, $S_{q}(0)=S_{q 0} \geq 0, E_{q}(0)=E_{q 0} \geq 0, I_{q}(0)=I_{q 0} \geq 0$ and $D_{t}^{\alpha}$ is the Caputo-Fabrizio fractional operator of order $\alpha$.

The total population $S(t)+E(t)+I(t)+R(t)+S_{q}(t)+E_{q}(t)+I_{q}(t)=N(t)$. Then

$$
\begin{aligned}
D_{t}^{\alpha} N(t) & =D_{t} S(t)+D_{t} E(t)+D_{t} I(t)+D_{t} R(t)+D_{t} S_{q}(t)+D_{t} E_{q}(t)+D_{t} I_{q}(t), \\
& \Rightarrow D_{t}^{\alpha} N(t)=\Lambda-\mu N(t)-\mu_{I}\left(I+I_{q}\right) .
\end{aligned}
$$

In the absence of the disease, $D_{t}^{\alpha} N(t)=\Lambda-\mu N(t)$, this shows that the population size $\mathrm{N}$ tends to carrying capacity

$$
\frac{\Lambda}{\mu} \text { as } t \rightarrow \infty
$$

\section{Quarantine Reproductive Number and Existence of Equilibrium}

The epidemic model (2) has a family of disease-free equilibrium, obtained by setting the right hand side of the equations in (2) to zero, given by

$$
D F E=\left(S^{0}, E^{0}, I^{0}, R^{0}, S_{q}^{0}, E_{q}^{0}, I_{q}^{0}\right),
$$

where $E^{0}=I^{0}=R^{0}=E_{q}^{0}=I_{q}^{0}=0$ and

$$
S^{0}=\frac{\Lambda\left(\delta_{q}+\mu\right)}{\mu\left(\mu+\delta_{q}+\delta_{s}\right)} \quad, \quad S_{q}^{0}=\frac{\Lambda \delta_{s}}{\mu\left(\mu+\delta_{q}+\delta_{s}\right)} .
$$

Now using the notation in [56], the non-negative matrix, $F$, for the new infection terms and the non-singular $M$-matrix, $V$, for the remaining transfer terms are given by

$$
\begin{aligned}
F & =\left(\begin{array}{llll}
q \beta S & \beta S & 0 & 0 \\
0 & 0 & 0 & 0 \\
0 & 0 & 0 & 0 \\
0 & 0 & 0 & 0
\end{array}\right) . \\
V & =\left(\begin{array}{llll}
\sigma_{0}+\sigma_{1}+\mu & 0 & 0 & 0 \\
-\sigma_{0} & \gamma_{0}+\gamma_{1}+\mu+\mu_{I} & 0 & 0 \\
-\sigma_{1} & 0 & q_{e}+\mu & 0 \\
0 & -\gamma_{1} & -q_{e} & q_{i}+\mu+\mu_{I}
\end{array}\right) .
\end{aligned}
$$

Thus

$$
F V^{-1}=\left(\begin{array}{cccc}
A & B & 0 & 0 \\
0 & 0 & 0 & 0 \\
0 & 0 & 0 & 0 \\
0 & 0 & 0 & 0
\end{array}\right)
$$


where

$$
A=\frac{q \beta S^{0}}{\sigma_{0}+\sigma_{1}+\mu}+\frac{\beta S^{0} \sigma_{0}}{\left(\sigma_{0}+\sigma_{1}+\mu\right)\left(\gamma_{0}+\gamma_{1}+\mu+\mu_{I}\right)} \quad \text { and } B=\frac{\beta S^{0}}{\gamma_{0}+\gamma_{1}+\mu+\mu_{I}} .
$$

The effective reproduction number (or quarantine-isolation reproduction number), denoted by $\mathcal{R}_{e}$, is given by $\mathcal{R}_{e}=\rho\left(F V^{-1}\right)$ where $\rho$ denotes the spectral radius. It follows that

$$
\mathcal{R}_{e}=\frac{\beta S^{0}\left[q\left(\gamma_{0}+\gamma_{1}+\mu+\mu_{I}\right)+\sigma_{0}\right]}{\left(\sigma_{0}+\sigma_{1}+\mu\right)\left(\gamma_{0}+\gamma_{1}+\mu+\mu_{I}\right)} .
$$

The system (2) has a unique endemic steady state

$$
E S S=\left(S^{*}, E^{*}, I^{*}, R^{*}, S_{q}^{*}, E_{q}^{*}, I_{q}^{*}\right)
$$

where

$$
\begin{aligned}
S^{*} & =\frac{S^{0}}{R_{e}}, \quad S_{q}=\frac{\delta_{s}}{\delta_{q}+\mu} S^{*}, \quad E^{*}=\frac{\Lambda\left(\delta_{q}+\mu\right)-\mu\left(\mu+\delta_{s}+\delta_{q}\right)}{\left(\sigma_{0}+\sigma_{1}+\mu\right)\left(\delta_{q}+\delta_{s}+\mu\right)} \frac{\Lambda}{R_{e}}, \\
E_{q} & =\frac{\sigma_{1}}{q_{e}+\mu} E^{*}, \quad I^{*}=\frac{\sigma}{\left(\gamma_{0}+\gamma_{1}+\mu+\mu_{I}\right)} E^{*} ; \\
I_{q}^{*} & =\frac{q_{e} \sigma_{1}\left(\gamma_{0}+\gamma_{1}+\mu+\mu_{I}\right)+\gamma_{1} \sigma_{0}\left(q_{e}+\mu\right)}{\left(q_{i}+\mu+\mu_{I}\right)\left(q_{e}+\mu\right)\left(\gamma_{0}+\gamma_{1}+\mu+\mu_{I}\right)} E^{*} .
\end{aligned}
$$

\section{Existence and Uniqueness of a System of Solutions}

In this section, we prove the existence of the system of solutions by applying the fixed-point theorem.

Let $\mathcal{H}=(C(J))^{7}$, and $C(J)$ be a Banach space of continuous $J \subset \mathbb{R} \rightarrow \mathbb{R}$ valued functions on the interval $J$ with the norm

$$
\left\|\left(S, E, I, R, S_{q}, E_{q}, I_{q}\right)\right\|=\|S\|+\|E\|+\|I\|+\|R\|+\left\|S_{q}\right\|+\left\|E_{q}\right\|+\left\|I_{q}\right\|,
$$

where $\|$.$\| denote the supremum norm in C(J)$.

Now using the integral operator of fractional order introduced by Losada and Nieto Jorge and Juan [32] on the system (2), we get

sleectfont

$$
\begin{aligned}
S(t)-S(0)= & \frac{2(1-\alpha)}{(2-\alpha) M(\alpha)}\left\{\Lambda-\beta S(t)(I(t)+q E(t))+\delta_{q} S_{q}(t)-\left(\delta_{s}+\mu\right) S(t)\right\} \\
& +\frac{2 \alpha}{(2-\alpha) M(\alpha)} \int_{0}^{t}\left\{\Lambda-\beta S(x)(I(x)+q E(x))+\delta_{q} S_{q}(x)-\left(\delta_{s}+\mu\right) S(x)\right\} d x, \\
E(t)-E(0)= & \frac{2(1-\alpha)}{(2-\alpha) M(\alpha)}\left\{\beta S(t)(I(t)+q E(t))-\left(\sigma_{0}+\sigma_{1}+\mu\right) E(t)\right\} \\
& +\frac{2 \alpha}{(2-\alpha) M(\alpha)} \int_{0}^{t}\left\{\beta S(x)(I(x)+q E(x))-\left(\sigma_{0}+\sigma_{1}+\mu\right) E(x)\right\} d x, \\
I(t)-I(0)= & \frac{2(1-\alpha)}{(2-\alpha) M(\alpha)}\left\{\sigma_{0} E(t)-\left(\gamma_{0}+\gamma_{1}+\mu+\mu_{I}\right) I(t)\right\} \\
& +\frac{2 \alpha}{(2-\alpha) M(\alpha)} \int_{0}^{t}\left\{\sigma_{0} E(x)-\left(\gamma_{0}+\gamma_{1}+\mu+\mu_{I}\right) I(x)\right\} d x, \\
R(t)-R(0)= & \frac{2(1-\alpha)}{(2-\alpha) M(\alpha)}\left\{\gamma_{0} I(t)+q_{i} I_{q}(t)-\mu R(t)\right\},
\end{aligned}
$$




$$
\begin{aligned}
& +\frac{2 \alpha}{(2-\alpha) M(\alpha)} \int_{0}^{t}\left\{\gamma_{0} I(x)+q_{i} I_{q}(x)-\mu R(x)\right\} d x, \\
S_{q}(t)-S_{q}(0)= & \frac{2(1-\alpha)}{(2-\alpha) M(\alpha)}\left\{\delta_{s} S(t)-\left(\delta_{q}+\mu\right) S_{q}(t)\right\} \\
& +\frac{2 \alpha}{(2-\alpha) M(\alpha)} \int_{0}^{t}\left\{\delta_{s} S(x)-\left(\delta_{q}+\mu\right) S_{q}(x)\right\} d x, \\
E_{q}(t)-E_{q}(0)= & \frac{2(1-\alpha)}{(2-\alpha) M(\alpha)}\left\{\sigma_{1} E(t)-\left(q_{e}+\mu\right) E_{q}(t)\right\} \\
& +\frac{2 \alpha}{(2-\alpha) M(\alpha)} \int_{0}^{t}\left\{\sigma_{1} E(x)-\left(q_{e}+\mu\right) E_{q}(x)\right\} d x, \\
I_{q}(t)-I_{q}(0)= & \frac{2(1-\alpha)}{(2-\alpha) M(\alpha)}\left\{q_{e} E_{q}(t)+\gamma_{1} I(t)-\left(q_{i}+\mu+\mu_{I}\right) I_{q}(t)\right\} \\
& +\frac{2 \alpha}{(2-\alpha) M(\alpha)} \int_{0}^{t}\left\{q_{e} E_{q}(x)+\gamma_{1} I(x)-\left(q_{i}+\mu+\mu_{I}\right) I_{q}(x)\right\} d x .
\end{aligned}
$$

For simplicity,

$$
\begin{aligned}
& \Phi_{1}(t, S)=\Lambda-\beta S(t)(I(t)+q E(t))+\delta_{q} S_{q}(t)-\left(\delta_{s}+\mu\right) S(t), \\
& \Phi_{2}(t, E)=\beta S(t)(I(t)+q E(t))-\left(\sigma_{0}+\sigma_{1}+\mu\right) E(t), \\
& \Phi_{3}(t, I)=\sigma_{0} E(t)-\left(\gamma_{0}+\gamma_{1}+\mu+\mu_{I}\right) I(t), \\
& \Phi_{4}(t, R)=\gamma_{0} I(t)+q_{i} I_{q}(t)-\mu R(t), \\
& \Phi_{5}\left(t, S_{q}\right)=\delta_{s} S(t)-\left(\delta_{q}+\mu\right) S_{q}(t), \\
& \Phi_{6}\left(t, E_{q}\right)=\sigma_{1} E(t)-\left(q_{e}+\mu\right) E_{q}(t), \\
& \Phi_{7}\left(t, I_{q}\right)=q_{e} E_{q}(t)+\gamma_{1} I(t)-\left(q_{i}+\mu+\mu_{I}\right) I_{q}(t) .
\end{aligned}
$$

To prove the following theorems, we will assume that $\|S(t)\| \leq c_{1},\|E(t)\| \leq c_{2},\|I(t)\| \leq$ $c_{3},\|R(t)\| \leq c_{4},\left\|S_{q}(t)\right\| \leq c_{5},\left\|E_{q}(t)\right\| \leq c_{6}$, and $\left\|I_{q}(t)\right\| \leq c_{7}$ where $c_{i}, i=1, \ldots, 7$, are some positive constants. Denote

$$
\begin{aligned}
& L_{1}=\beta c_{3}+q \beta c_{2}+\delta_{s}+\mu, \quad L_{2}=q \beta c_{1}+\sigma_{0}+\sigma_{1}+\mu, \\
& L_{3}=\gamma_{0}+\gamma_{1}+\mu+\mu_{I}, \quad L_{4}=\mu, \quad L_{5}=\delta_{s}+\mu, \quad L_{6}=q_{e}+\mu, \quad L_{7}=q_{i}+\mu+\mu_{I} .
\end{aligned}
$$

Theorem 3 The kernels $\Phi_{i}(i=1, \ldots, 7)$, satisfy the Lipschitz condition and contraction if the inequality given below holds

$$
0 \leq L_{i}<1, \text { for } i=1, \ldots 7 .
$$

Proof Let $S_{1}$ and $S_{2}$ be two functions, then

$$
\begin{aligned}
\left\|\Phi_{1}\left(t, S_{1}\right)-\Phi_{1}\left(t, S_{2}\right)\right\| & =\left\|\left(-\beta I(t)-q \beta E(t)-\left(\delta_{s}+\mu\right)\right)\left(S_{1}-S_{2}\right)\right\| \\
& \leq\left[\beta\|I\|+q \beta\|E\|+\left(\delta_{s}+\mu\right)\right]\left\|S_{1}(t)-S_{2}(t)\right\| \\
& \leq\left(\beta c_{3}+q \beta c_{2}+\delta_{s}+\mu\right)\left\|S_{1}(t)-S_{2}(t)\right\| .
\end{aligned}
$$

Thus

$$
\left\|\Phi_{1}\left(t, S_{1}\right)-\Phi_{1}\left(t, S_{2}\right)\right\| \leq L_{1}\left\|S_{1}(t)-S_{2}(t)\right\| .
$$

Hence, for $\Phi_{1}$ the Lipschitz condition is obtained. Similarly for $\Phi_{2}, \Phi_{3}, \Phi_{4}, \Phi_{5}, \Phi_{6}$, and $\Phi_{7}$ the Lipschiz condition can be easily verified and given below:

$$
\left\|\Phi_{2}\left(t, E_{1}\right)-\Phi_{2}\left(t, E_{2}\right)\right\| \leq L_{2}\left\|E_{1}(t)-E_{2}(t)\right\|,
$$




$$
\begin{aligned}
& \left\|\Phi_{3}\left(t, I_{1}\right)-\Phi_{3}\left(t, I_{2}\right)\right\| \leq L_{3}\left\|I_{1}(t)-I_{2}(t)\right\|, \\
& \left\|\Phi_{4}\left(t, R_{1}\right)-\Phi_{4}\left(t, R_{2}\right)\right\| \leq L_{4}\left\|R_{1}(t)-R_{2}(t)\right\|, \\
& \left\|\Phi_{5}\left(t, S_{q 1}\right)-\Phi_{5}\left(t, S_{q 2}\right)\right\| \leq L_{5}\left\|S_{q 1}(t)-S_{q 2}(t)\right\|, \\
& \left\|\Phi_{6}\left(t, E_{q 1}\right)-\Phi_{6}\left(t, E_{q 2}\right)\right\| \leq L_{6}\left\|E_{q 1}(t)-E_{q 2}(t)\right\|, \\
& \left\|\Phi_{7}\left(t, I_{q 1}\right)-\Phi_{7}\left(t, I_{q 2}\right)\right\| \leq L_{7}\left\|I_{q 1}(t)-I_{q 2}(t)\right\|,
\end{aligned}
$$

Now we write the system (2) in the following recursive form:

$$
\left\{\begin{array}{l}
S_{n}(t)=\frac{2(1-\alpha)}{(2-\alpha) M(\alpha)} \Phi_{1}\left(t, S_{n-1}\right)+\frac{2 \alpha}{(2-\alpha) M(\alpha)} \int_{0}^{t} \Phi_{1}\left(x, S_{n-1}\right) \mathrm{d} x, \\
E_{n}(t)=\frac{2(1-\alpha)}{(2-\alpha) M(\alpha)} \Phi_{2}\left(t, E_{n-1}\right)+\frac{2 \alpha}{(2-\alpha) M(\alpha)} \int_{0}^{t} \Phi_{2}\left(x, E_{n-1}\right) \mathrm{d} x \\
I_{n}(t)=\frac{2(1-\alpha)}{(2-\alpha) M(\alpha)} \Phi_{3}\left(t, I_{n-1}\right)+\frac{2 \alpha}{(2-\alpha) M(\alpha)} \int_{0}^{t} \Phi_{3}\left(x, I_{n-1}\right) \mathrm{d} x \\
R_{n}(t)=\frac{2(1-\alpha)}{(2-\alpha) M(\alpha)} \Phi_{4}\left(t, R_{n-1}\right)+\frac{2 \alpha}{(2-\alpha) M(\alpha)} \int_{0}^{t} \Phi_{4}\left(x, R_{n-1}\right) \mathrm{d} x \\
S_{q, n}(t)=\frac{2(1-\alpha)}{(2-\alpha) M(\alpha)} \Phi_{5}\left(t, S_{q, n-1}\right)+\frac{2 \alpha}{(2-\alpha) M(\alpha)} \int_{0}^{t} \Phi_{5}\left(x, S_{q, n-1}\right) \mathrm{d} x \\
E_{q, n}(t)=\frac{2(1-\alpha)}{(2-\alpha) M(\alpha)} \Phi_{6}\left(t, E_{q, n-1}\right)+\frac{2 \alpha}{(2-\alpha) M(\alpha)} \int_{0}^{t} \Phi_{6}\left(x, E_{q, n-1}\right) \mathrm{d} x \\
I_{q, n}(t)=\frac{2(1-\alpha)}{(2-\alpha) M(\alpha)} \Phi_{7}\left(t, I_{q, n-1}\right)+\frac{2 \alpha}{(2-\alpha) M(\alpha)} \int_{0}^{t} \Phi_{7}\left(x, I_{q, n-1}\right) \mathrm{d} x .
\end{array}\right.
$$

The initial conditions are

$S_{0}(t)=S(0), E_{0}(t)=E(0), I_{0}(t)=(0), R_{0}(t)=R(0), S_{q, 0}(t)=S_{q}(0), E_{q, 0}(t)=E_{q}(0)$ and $I_{q, 0}=I_{q}(0)$.

Then we get the following expressions for the difference between the successive terms:

$$
\begin{aligned}
W_{n}^{1}(t)= & S_{n}(t)-S_{n-1}(t)=\frac{2(1-\alpha)}{(2-\alpha) M(\alpha)}\left(\Phi_{1}\left(t, S_{n-1}\right)-\Phi_{1}\left(t, S_{n-2}\right)\right) \\
& +\frac{2 \alpha}{(2-\alpha) M(\alpha)} \int_{0}^{t}\left(\Phi_{1}\left(x, S_{n-1}\right)-\Phi_{1}\left(x, S_{n-2}\right)\right) d x, \\
W_{n}^{2}(t)= & E_{n}(t)-E_{n-1}(t)=\frac{2(1-\alpha)}{(2-\alpha) M(\alpha)}\left(\Phi_{2}\left(t, E_{n-1}\right)-\Phi_{2}\left(t, E_{n-2}\right)\right) \\
& +\frac{2 \alpha}{(2-\alpha) M(\alpha)} \int_{0}^{t}\left(\Phi_{2}\left(x, E_{n-1}\right)-\Phi_{2}\left(x, E_{n-2}\right)\right) d x, \\
W_{n}^{3}(t)= & I_{n}(t)-I_{n-1}(t)=\frac{2(1-\alpha)}{(2-\alpha) M(\alpha)}\left(\Phi_{3}\left(t, I_{n-1}\right)-\Phi_{3}\left(t, I_{n-2}\right)\right) \\
& +\frac{2 \alpha}{(2-\alpha) M(\alpha)} \int_{0}^{t}\left(\Phi_{3}\left(x, I_{n-1}\right)-\Phi_{3}\left(x, I_{n-2}\right)\right) d x, \\
W_{n}^{4}(t)= & R_{n}(t)-R_{n-1}(t)=\frac{2(1-\alpha)}{(2-\alpha) M(\alpha)}\left(\Phi_{4}\left(t, R_{n-1}\right)-\Phi_{4}\left(t, R_{n-2}\right)\right) \\
& +\frac{2 \alpha}{(2-\alpha) M(\alpha)} \int_{0}^{t}\left(\Phi_{4}\left(x, R_{n-1}\right)-\Phi_{4}\left(x, R_{n-2}\right)\right) d x, \\
W_{n}^{5}(t)= & S_{q, n}(t)-S_{n-1}(t)=\frac{2(1-\alpha)}{(2-\alpha) M(\alpha)}\left(\Phi_{5}\left(t, S_{q, n-1}\right)-\Phi_{5}\left(t, S_{q, n-2}\right)\right) \\
& +\frac{2 \alpha}{(2-\alpha) M(\alpha)} \int_{0}^{t}\left(\Phi_{5}\left(x, S_{q, n-1}\right)-\Phi_{5}\left(x, S_{q, n-2}\right)\right) d x,
\end{aligned}
$$




$$
\begin{aligned}
W_{n}^{6}(t)= & E_{q, n}(t)-E_{q, n-1}(t)=\frac{2(1-\alpha)}{(2-\alpha) M(\alpha)}\left(\Phi_{6}\left(t, E_{q, n-1}\right)-\Phi_{6}\left(t, E_{q, n-2}\right)\right) \\
& +\frac{2 \alpha}{(2-\alpha) M(\alpha)} \int_{0}^{t}\left(\Phi_{6}\left(x, E_{q, n-1}\right)-\Phi_{6}\left(x, E_{q, n-2}\right)\right) d x, \\
W_{n}^{7}(t) & =I_{q, n}(t)-I_{q, n-1}(t)=\frac{2(1-\alpha)}{(2-\alpha) M(\alpha)}\left(\Phi_{7}\left(t, I_{q, n-1}\right)-\Phi_{7}\left(t, I_{q, n-2}\right)\right) \\
& +\frac{2 \alpha}{(2-\alpha) M(\alpha)} \int_{0}^{t}\left(\Phi_{7}\left(x, I_{q, n-1}\right)-\Phi_{7}\left(x, I_{q, n-2}\right)\right) d x .
\end{aligned}
$$

Taking the norm on both side of the Eq. (11), applying triangular inequality and using the Lipschitz condition proved in (9) we obtained

$$
\begin{aligned}
\left\|W_{n}^{1}(t)\right\| & =\left\|S_{n}(t)-S_{n-1}(t)\right\| \\
& \leq \frac{2(1-\alpha)}{(2-\alpha) M(\alpha)} L_{1}\left\|S_{n-1}-S_{n-2}\right\|+\frac{2 \alpha}{(2-\alpha) M(\alpha)} L_{1} \int_{0}^{t}\left\|S_{n-1}-S_{n-2}\right\| d x .
\end{aligned}
$$

Then, we have

$$
\left\|W_{n}^{1}(t)\right\| \leq \frac{2(1-\alpha)}{(2-\alpha) M(\alpha)} L_{1}\left\|W_{n-1}^{1}(t)\right\|+\frac{2 \alpha}{(2-\alpha) M(\alpha)} L_{1} \int_{0}^{t}\left\|W_{n-1}^{1}(x)\right\| d x
$$

Similarly, for the remaining equations of the system, we obtain the following results:

$$
\begin{aligned}
\left\|W_{n}^{2}(t)\right\| & \leq \frac{2(1-\alpha)}{(2-\alpha) M(\alpha)} L_{2}\left\|W_{n-1}^{2}(t)\right\|+\frac{2 \alpha}{(2-\alpha) M(\alpha)} L_{2} \int_{0}^{t}\left\|W_{n-1}^{2}(x)\right\| d x, \\
\left\|W_{n}^{3}(t)\right\| & \leq \frac{2(1-\alpha)}{(2-\alpha) M(\alpha)} L_{3}\left\|W_{n-1}^{3}(t)\right\|+\frac{2 \alpha}{(2-\alpha) M(\alpha)} L_{3} \int_{0}^{t}\left\|W_{n-1}^{3}(x)\right\| d x, \\
\left\|W_{n}^{4}(t)\right\| & \leq \frac{2(1-\alpha)}{(2-\alpha) M(\alpha)} L_{4}\left\|W_{n-1}^{4}(t)\right\|+\frac{2 \alpha}{(2-\alpha) M(\alpha)} L_{4} \int_{0}^{t}\left\|W_{n-1}^{4}(x)\right\| d x, \\
\left\|W_{n}^{5}(t)\right\| & \leq \frac{2(1-\alpha)}{(2-\alpha) M(\alpha)} L_{5}\left\|W_{n-1}^{5}(t)\right\|+\frac{2 \alpha}{(2-\alpha) M(\alpha)} L_{5} \int_{0}^{t}\left\|W_{n-1}^{5}(x)\right\| d x, \\
\left\|W_{n}^{6}(t)\right\| & \leq \frac{2(1-\alpha)}{(2-\alpha) M(\alpha)} L_{6}\left\|W_{n-1}^{6}(t)\right\|+\frac{2 \alpha}{(2-\alpha) M(\alpha)} L_{6} \int_{0}^{t}\left\|W_{n-1}^{6}(x)\right\| d x, \\
\left\|W_{n}^{7}(t)\right\| & \leq \frac{2(1-\alpha)}{(2-\alpha) M(\alpha)} L_{7}\left\|W_{n-1}^{7}(t)\right\|+\frac{2 \alpha}{(2-\alpha) M(\alpha)} L_{7} \int_{0}^{t}\left\|W_{n-1}^{7}(x)\right\| d x,
\end{aligned}
$$

Immediately, in view of the above results, we state the following theorem.

Theorem 4 The solution of the CF-fractional model given in (2) will exist and be unique if we can find some $t_{0}$ such that

$$
\frac{2(1-\alpha)}{(2-\alpha) M(\alpha)} L_{i}+\frac{2 \alpha t_{0}}{(2-\alpha) M(\alpha)} L_{i}<1, \text { for } i=1,2, \ldots, 7
$$

Proof As the functions $S(t), E(t), I(t), R(t), S_{q}(t), I_{q}(t)$ and $E_{q}(t)$ are bounded and fulfill Lipschitz condition. So, by considering Eqs. (18)-(24), we obtain the following relations: 


$$
\begin{aligned}
& \left\|W_{n}^{1}(t)\right\| \leq\left\|S_{n}(0)\right\|\left[\frac{2(1-\alpha)}{(2-\alpha) M(\alpha)} L_{1}+\frac{2 \alpha t_{0}}{(2-\alpha) M(\alpha)} L_{1}\right]^{n}, \\
& \left\|W_{n}^{2}(t)\right\| \leq\left\|E_{n}(0)\right\|\left[\frac{2(1-\alpha)}{(2-\alpha) M(\alpha)} L_{2}+\frac{2 \alpha t_{0}}{(2-\alpha) M(\alpha)} L_{2}\right]^{n}, \\
& \left\|W_{n}^{3}(t)\right\| \leq\left\|I_{n}(0)\right\|\left[\frac{2(1-\alpha)}{(2-\alpha) M(\alpha)} L_{3}+\frac{2 \alpha t_{0}}{(2-\alpha) M(\alpha)} L_{3}\right]^{n}, \\
& \left\|W_{n}^{4}(t)\right\| \leq\left\|R_{n}(0)\right\|\left[\frac{2(1-\alpha)}{(2-\alpha) M(\alpha)} L_{4}+\frac{2 \alpha t_{0}}{(2-\alpha) M(\alpha)} L_{4}\right]^{n}, \\
& \left\|W_{n}^{5}(t)\right\| \leq\left\|S_{q, n}(0)\right\|\left[\frac{2(1-\alpha)}{(2-\alpha) M(\alpha)} L_{5}+\frac{2 \alpha t_{0}}{(2-\alpha) M(\alpha)} L_{5}\right]^{n}, \\
& \left\|W_{n}^{6}(t)\right\| \leq\left\|E_{q, n}(0)\right\|\left[\frac{2(1-\alpha)}{(2-\alpha) M(\alpha)} L_{6}+\frac{2 \alpha t_{0}}{(2-\alpha) M(\alpha)} L_{6}\right]^{n}, \\
& \left\|W_{n}^{7}(t)\right\| \leq\left\|I_{q, n}(0)\right\|\left[\frac{2(1-\alpha)}{(2-\alpha) M(\alpha)} L_{7}+\frac{2 \alpha t_{0}}{(2-\alpha) M(\alpha)} L_{7}\right]^{n} .
\end{aligned}
$$

Thus, the proof the existence and continuity of the solutions is completed. To show that the above functions are solutions of system (2), we set as follows:

$$
\begin{gathered}
S(t)-S(0)=S_{n}(t)-B_{n}^{1}(t), \\
E(t)-E(0)=E_{n}(t)-B_{n}^{2}(t), \\
I(t)-I(0)=I_{n}(t)-B_{n}^{3}(t), \\
R(t)-R(0)=R_{n}(t)-B_{n}^{4}(t), \\
S_{q}(t)-S_{q}(0)=S_{q, n}(t)-B_{n}^{5}(t), \\
E_{q}(t)-E_{q}(0)=E_{q, n}(t)-B_{n}^{6}(t), \\
I_{q}(t)-I_{q}(0)=S_{q, n}(t)-B_{n}^{7}(t) .
\end{gathered}
$$

Therefore, we have

$$
\begin{aligned}
\left\|B_{n}^{1}(t)\right\| \leq & \frac{2(1-\alpha)}{(2-\alpha) M(\alpha)}\left\|\left(\Phi_{1}(t, S)-\Phi_{1}\left(t, S_{n-1}\right)\right)\right\| \\
& +\frac{2 \alpha}{(2-\alpha) M(\alpha)} \int_{0}^{t}\left\|\left(\Phi_{1}(y, S)-\Phi_{1}\left(y, S_{n-1}\right)\right)\right\| d y, \\
\leq & \frac{2(1-\alpha)}{(2-\alpha) M(\alpha)} L_{1}\left\|S-S_{n-1}\right\|+\frac{2 \alpha t}{(2-\alpha) M(\alpha)} L_{1}\left\|S-S_{n-1}\right\| .
\end{aligned}
$$

On using this process recursively, it yields

$$
\left\|B_{n}^{1}(t)\right\| \leq\left(\frac{2(1-\alpha)}{(2-\alpha) M(\alpha)}+\frac{2 \alpha}{(2-\alpha) M(\alpha)} t\right)^{n+1} L_{1}^{n+1} c_{1} .
$$

where $c_{1}$ is a positive constant. Then at $t_{0}$, we have

$$
\left\|B_{n}^{1}(t)\right\| \leq\left(\frac{2(1-\alpha)}{(2-\alpha) M(\alpha)}+\frac{2 \alpha t_{0}}{(2-\alpha) M(\alpha)}\right)^{n+1} L_{1}^{n+1} c_{1} .
$$

Now taking the limit as $n$ tends to infinity, we get

$$
\left\|B_{n}^{1}(t)\right\| \rightarrow 0 \text {. }
$$


Repeating the same procedure we obtain

$$
\left\|B_{n}^{i}(t)\right\| \rightarrow 0, \text { for } i=2, \ldots, 7 .
$$

Hence, proof of existence is verified.

Next to show the uniqueness of the solution of the model (2), suppose on the contrary that there exists another set of solutions $S_{1}(t), E_{1}(t), I_{1}(t), R_{1}(t), S_{q, 1}(t), E_{q, 1}(t)$ and $I_{q, 1}(t)$ then

$$
\begin{aligned}
S(t)-S_{1}(t)= & \frac{2(1-\alpha)}{(2-\alpha) M(\alpha)}\left(\Phi_{1}(t, S)-\Phi_{1}\left(t, S_{1}\right)\right) \\
& +\frac{2 \alpha}{(2-\alpha) M(\alpha)} \int_{0}^{t}\left(\Phi_{1}(y, S)-\Phi_{1}\left(y, S_{1}\right)\right) d y .
\end{aligned}
$$

Applying norm in the above equation, we get

$$
\begin{aligned}
\left\|S(t)-S_{1}(t)\right\| \leq & \frac{2(1-\alpha)}{(2-\alpha) M(\alpha)}\left\|\Phi_{1}(t, S)-\Phi_{1}\left(t, S_{1}\right)\right\| \\
& +\frac{2 \alpha}{(2-\alpha) M(\alpha)} \int_{0}^{t}\left\|\left(\Phi_{1}(y, S)-\Phi_{1}\left(y, S_{1}\right)\right)\right\| d y .
\end{aligned}
$$

By employing the Lipschitz condition of kernel, we have

$$
\begin{aligned}
\left\|S(t)-S_{1}(t)\right\| \leq & \frac{2(1-\alpha)}{(2-\alpha) M(\alpha)} L_{1}\left\|S(t)-S_{1}(t)\right\| \\
& +\frac{2 \alpha}{(2-\alpha) M(\alpha)} L_{1} t\left\|S(t)-S_{1}(t)\right\| .
\end{aligned}
$$

It gives

$$
\left\|S(t)-S_{1}(t)\right\|\left(1-\frac{2(1-\alpha)}{(2-\alpha) M(\alpha)} L_{1}-\frac{2 \alpha}{(2-\alpha) M(\alpha)} L_{1} t\right) \leq 0 .
$$

Clearly $S(t)=S_{1}(t)$ if

$$
1-\frac{2(1-\alpha)}{(2-\alpha) M(\alpha)} L_{1}-\frac{2 \alpha}{(2-\alpha) M(\alpha)} L_{1} t>0 .
$$

Employing the same procedure, we get

$E(t)=E_{1}(t), I(t)=I_{1}(t), R(t)=R_{1}(t), S_{q}(t)=S_{q, 1}(t), E_{q}(t)=E_{q, 1}(t)$ and $I_{q}(t)=I_{q, 1}(t)$.

Hence the solution is unique if condition (25) is satisfied.

\section{Numerical Scheme}

In this Section, we present a numerical solution of the fractional order model (2). Then The numerical simulations are obtained using the proposed scheme for Adams-Bashforth. For this purpose we use the fractional $m$-step Adams Bashforth method to approximate the CF-fractional integral operator and to obtain an iterative scheme,

Consider the Caputo-Fabrizio fractional differential equation

$$
\left\{\begin{array}{l}
{ }^{C F} D_{t}^{\alpha}(x(t))=f(t, x(t)), \quad 0<\alpha<1, \\
x(0)=x_{0} .
\end{array}\right.
$$


Now applying the fractional integral to system (28) we get:

$$
x(t)-x(0)=\frac{1-\alpha}{M(\alpha)} f(t, x(t))+\frac{\alpha}{M(\alpha)} \int_{0}^{t} f(\tau, x(\tau)) d \tau .
$$

At $t=t_{n+1}$, and $t=t_{n}$ for $n=1,2,3, \ldots$, we have

$$
x\left(t_{n+1}\right)-x(0)=\frac{1-\alpha}{M(\alpha)} f\left(t_{n}, x\left(t_{n}\right)\right)+\frac{\alpha}{M(\alpha)} \int_{0}^{t_{n+1}} f(\tau, x(\tau)) d \tau,
$$

and

$$
x\left(t_{n}\right)-x(0)=\frac{1-\alpha}{M(\alpha)} f\left(t_{n-1}, x\left(t_{n-1}\right)\right)+\frac{\alpha}{M(\alpha)} \int_{0}^{t_{n}} f(\tau, x(\tau)) d \tau .
$$

Subtracting (30) from (29), the following equation obtained

$$
x\left(t_{n+1}\right)-x\left(t_{n}\right)=\frac{1-\alpha}{M(\alpha)}\left\{f\left(t_{n}, x\left(t_{n}\right)\right)-f\left(t_{n-1}, x_{n-1}\right)\right\}+\frac{\alpha}{M(\alpha)} \int_{t_{n}}^{t_{n+1}} f(t, x(t)) d t .
$$

The function $f(t, x(t))$ can be approximated over $\left[t_{n}, t_{n+1}\right]$, for $n=0,1,2, \ldots$, and $h=t_{n+1}-t_{n}$ using the interpolation polynomial of Lagrange of degree $m$, we have

$$
f(t, x(t)) \approx P(t, x(t))=\sum_{j=n-m}^{n} L_{j}(t) f\left(t_{j}, x_{j}\right),
$$

where $L_{j}$ are the Lagrange functions on the $(m+1)$ points $\left\{t_{n-m}, \ldots, t_{n-1}, t_{n}\right\}$ and given by

$$
L_{j}(t)=\prod_{\substack{k=n-m \\ k \neq j}}^{n} \frac{t-t_{k}}{t_{j}-t_{k}}
$$

Therefore, the integral becomes

$$
\begin{aligned}
\int_{t_{n}}^{t_{n+1}} f(t, x(t)) d t & \approx \int_{t_{n+s-1}}^{t_{n+s}} P(t, x(t)) d t \\
& =\int_{t_{n}}^{t_{n+1}} \sum_{j=n-m}^{n} L_{j}(t) f\left(t_{j}, x_{j}\right) d t \\
& =\sum_{j=n-m}^{n} f\left(t_{j}, x_{j}\right) \int_{t_{n}}^{t_{n+1}} L_{j}(t) d t .
\end{aligned}
$$

Thus

$$
\begin{aligned}
x\left(t_{n+1}\right)-x\left(t_{n}\right)= & \frac{1-\alpha}{M(\alpha)}\left\{f\left(t_{n}, x\left(t_{n}\right)\right)-f\left(t_{n-1}, x_{n-1}\right)\right\} \\
& +\frac{\alpha}{M(\alpha)} \sum_{j=n-m}^{n} f\left(t_{j}, x_{j}\right) \int_{t_{n}}^{t_{n+1}} L_{j}(t) d t .
\end{aligned}
$$

This method is known as m-step Adams-Bashforth method, for $m=1$ we obtain the CF-fractional second-order Adams-Bashforth method (see also [11]) 


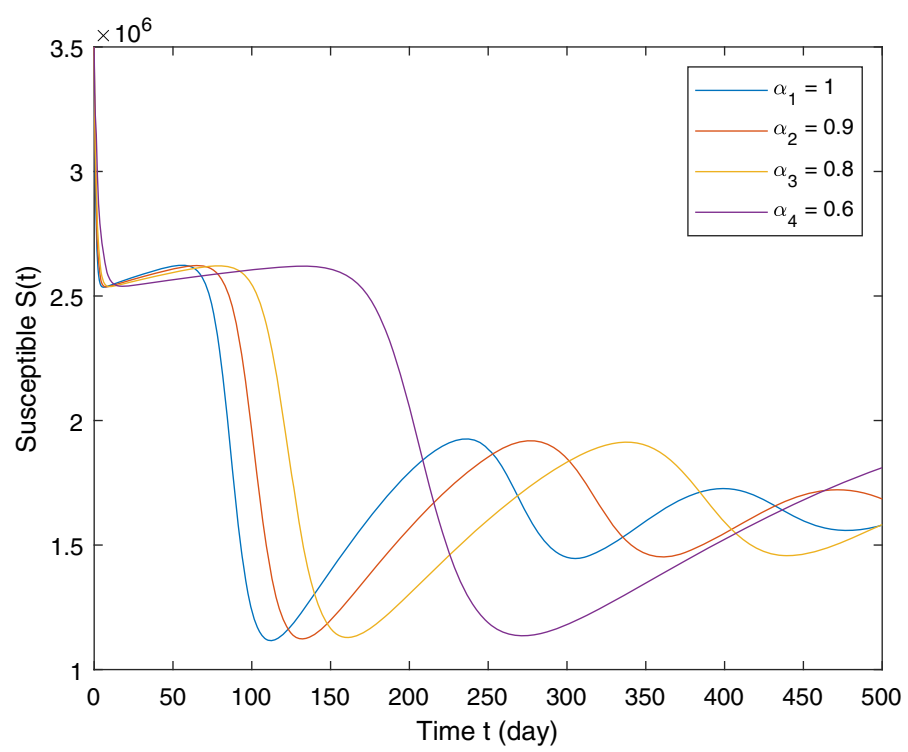

(a) Susceptible $S(t)$

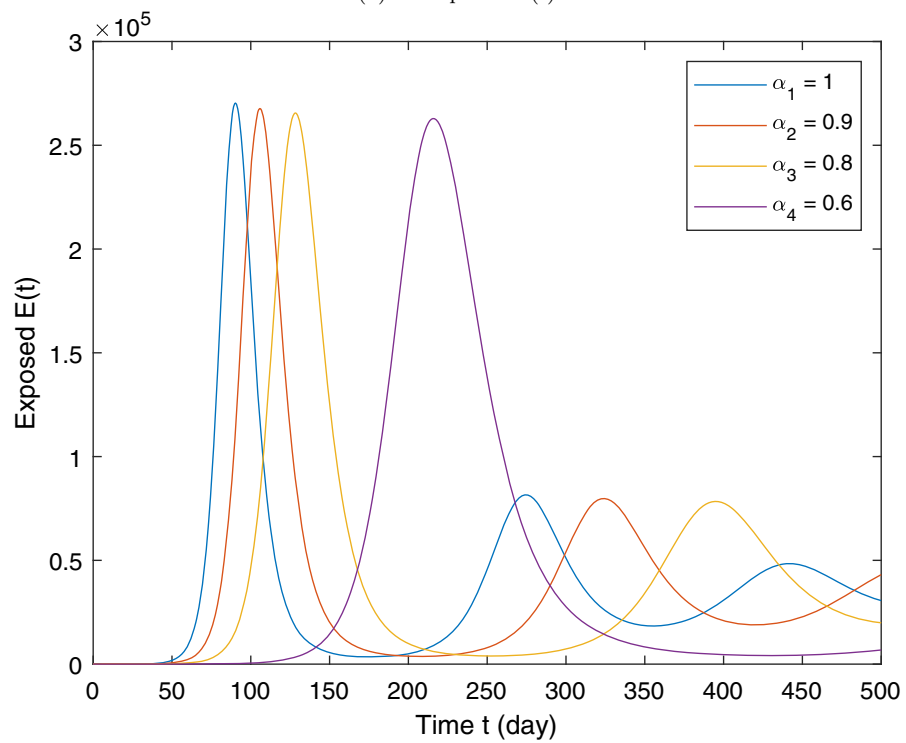

(b) Exposed $E(t)$

Fig. 2 Graphical representation of numerical solution for susceptible $S(t)$ and Exposed $E(t)$ at various fractional order of the considered model. Parameter values used are as given in Table 1

$$
x_{n+1}=x_{n}+\frac{2-2 \alpha+3 \alpha h}{2 M(\alpha)} f\left(t_{n}, x_{n}\right)+\frac{2-2 \alpha+\alpha h}{2 M(\alpha)} f\left(t_{n-1}, x_{n-1}\right) .
$$

For $m=2$ we obtain the CF-fractional three-order Adams-Bashforth method (see also Moore et al. [39]) 


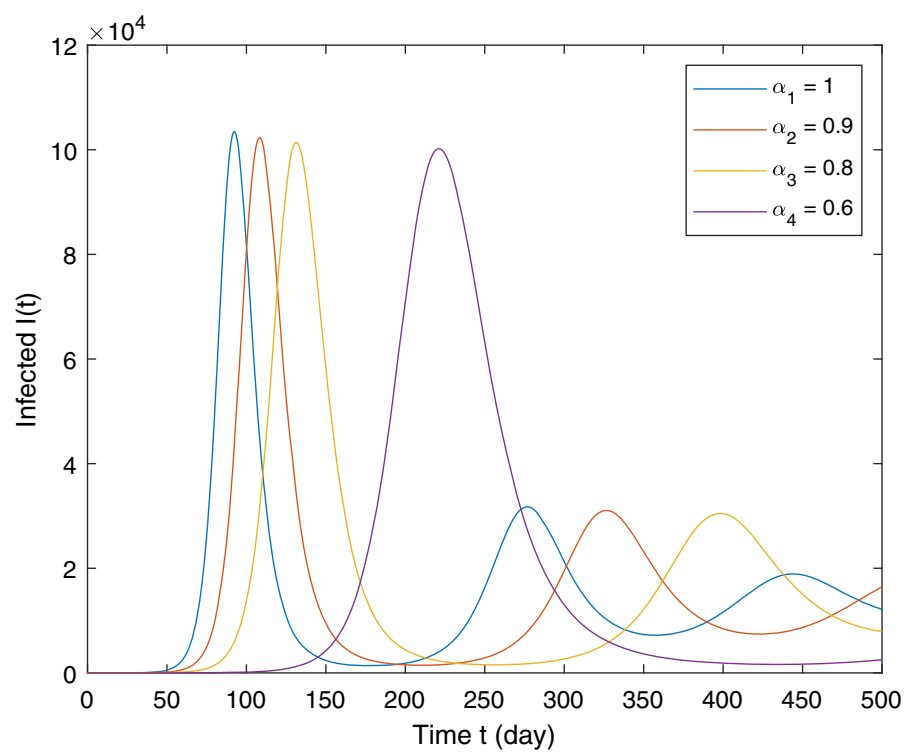

(a) Infected $I(t)$

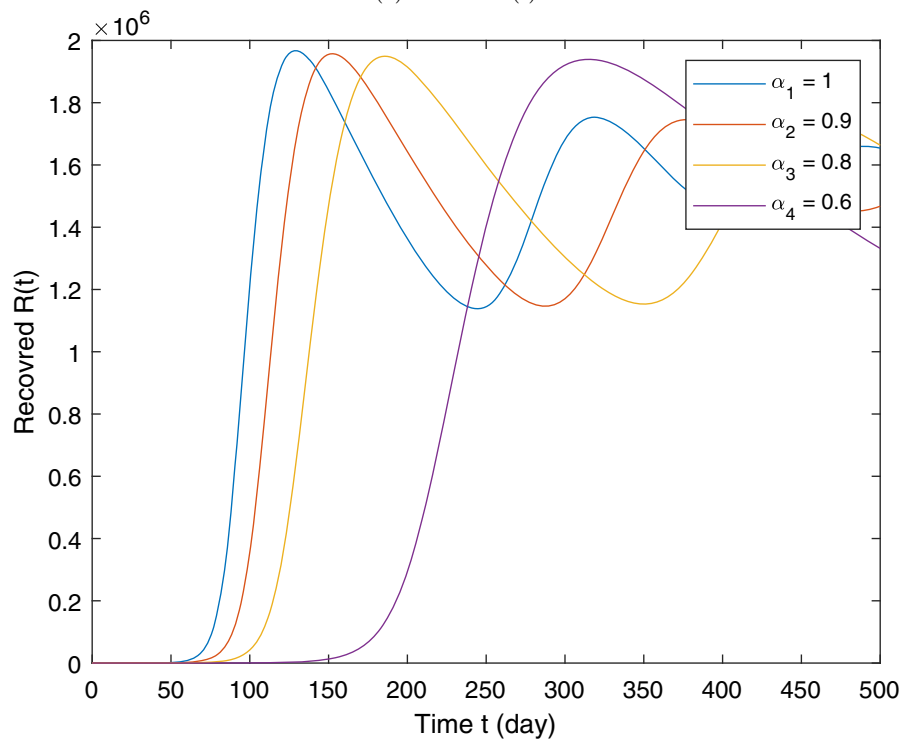

(b) Recovred $R(t)$

Fig. 3 Graphical representation of numerical solution for Infected $I(t)$ and Recovered $R(t)$ at various fractional order of the considered model. Parameter values used are as given in Table 1

$$
\begin{aligned}
x_{n+1}= & x_{n}+\left(\frac{1-\alpha}{M(\alpha)}+\frac{23 \alpha h}{12 M(\alpha)}\right) f\left(t_{n}, x_{n}\right)-\left(\frac{1-\alpha}{M(\alpha)}+\frac{16 \alpha h}{12 M(\alpha)}\right) f\left(t_{n-1}, x_{n-1}\right) \\
& +\frac{5 \alpha h}{12 M(\alpha)} f\left(t_{n-2}, x_{n-2}\right) .
\end{aligned}
$$




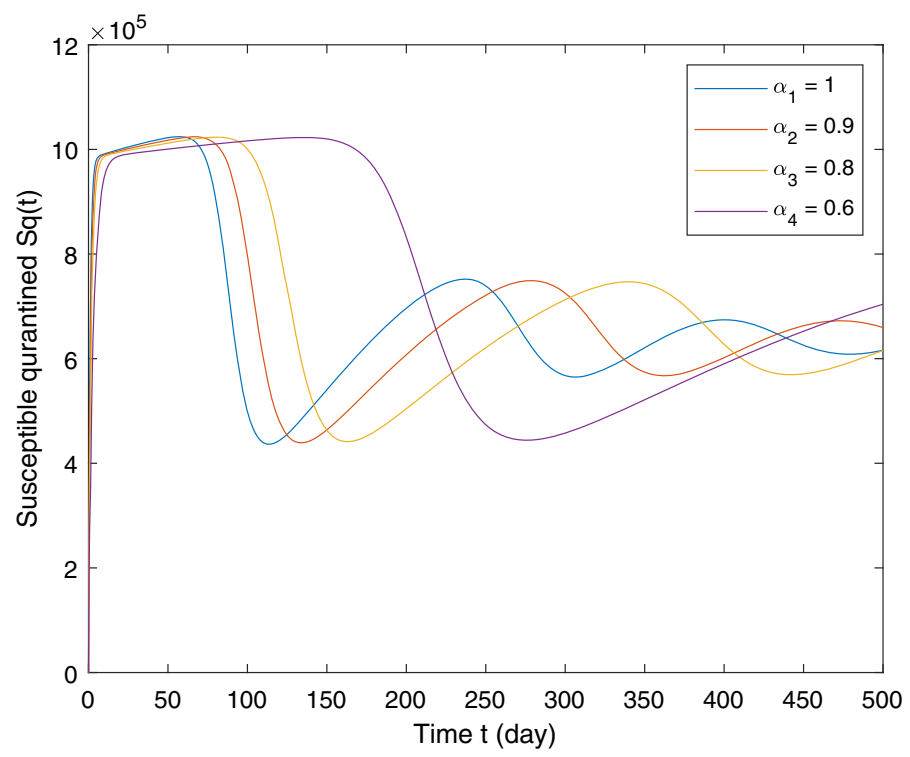

(a) Susceptible quarantined $S_{q}(t)$

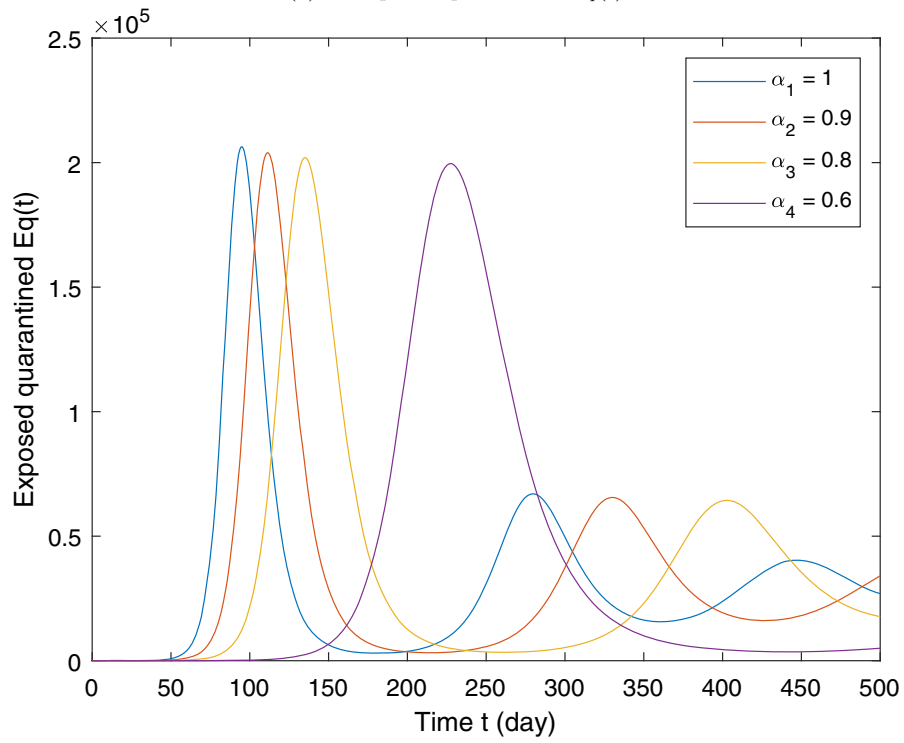

(b) Exposed quarantined $E_{q}(t)$

Fig. 4 Graphical representation of numerical solution for Susceptible quarantined $S_{q}(t)$ and Exposed quarantined $E_{q}(t)$ at various fractional order of the considered model. Parameter values used are as given in Table 1. The initial conditions are the same of the Section 7.2

For $m=3$ we obtain the CF-fractional four-order Adams-Bashforth method

$$
\begin{aligned}
x_{n+1}= & x_{n}+\left(\frac{1-\alpha}{M(\alpha)}+\frac{55 \alpha h}{24 M(\alpha)}\right) f\left(t_{n}, x_{n}\right)-\left(\frac{1-\alpha}{M(\alpha)}+\frac{59 \alpha h}{24 M(\alpha)}\right) f\left(t_{n-1}, x_{n-1}\right) \\
& +\frac{37 \alpha h}{24 M(\alpha)} f\left(t_{n-2}, x_{n-2}\right)-\frac{9 \alpha h}{24 M(\alpha)} f\left(t_{n-3}, x_{n-3}\right) .
\end{aligned}
$$




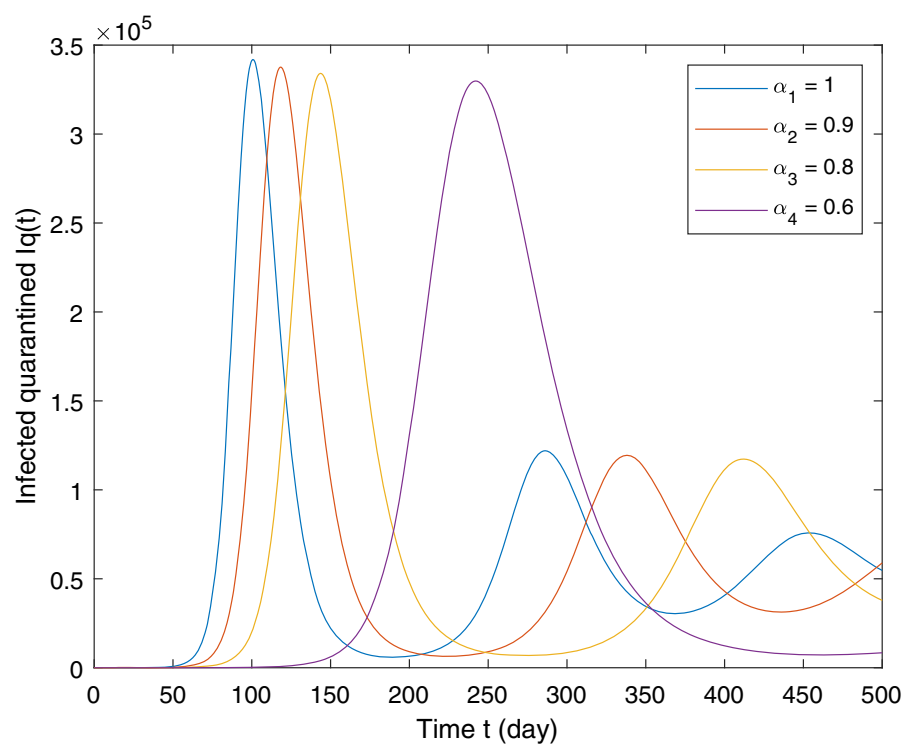

Fig. 5 Graphical representation of numerical solution for Infected isolated $I_{q}(t)$ at various fractional order of the considered model. Parameter values used are as given in Table 1. The initial conditions are the same of the Section 7.2

It should be noted that when $\alpha=1$ this method reduces to the classical Adams-Bashforth m-step method.

The truncation error for the m-step formula can be estimated by using the error estimate for the Lagrange interpolating polynomial, namely,

$$
\begin{aligned}
& f(t, x(t))=P_{m}(t)+E_{m}(t) \quad \text { where } \\
& E_{m}(t)=\prod_{j=n-m}^{n}\left(t-t_{j}\right) \frac{f^{(m+1)}\left(\xi_{n}, x\left(\xi_{n}\right)\right)}{(m+1) !} ; \quad \xi_{n} \in\left[t_{n-m}, t_{n}\right] .
\end{aligned}
$$

Then we have

$$
\int_{t_{n}}^{t_{n+1}} E_{m}(t) d t=\frac{f^{(m+1)}\left(\xi_{n}, x\left(\xi_{n}\right)\right)}{(m+1) !} \int_{t_{n}}^{t_{n+1}} \prod_{j=n-m}^{n}\left(t-t_{j}\right) d t
$$

a simple calculation shows that the last quantity is bounded by

$$
C_{m}=\frac{c h^{m+2}}{(m+1) !} .
$$

where $c$ is a constant only depends on $f$ and $m$.

Now using 4-step fractional Adams-Bashforth scheme to obtain numerical solutions of the fractional model (2) we get for the first equation in (2) the following scheme:

$$
\begin{aligned}
S_{n+1}= & S_{n}+\tilde{h}_{1}(\alpha) \Phi_{1}\left(t_{n}, S_{n}\right)+\tilde{h}_{2}(\alpha) \Phi_{1}\left(t_{n-1}, S_{n-1}\right)+\tilde{h}_{3}(\alpha) \Phi_{1}\left(t_{n-2}, S_{n-2}\right) \\
& +\tilde{h}_{4}(\alpha) \Phi_{1}\left(t_{n-3}, S_{n-3}\right) .
\end{aligned}
$$




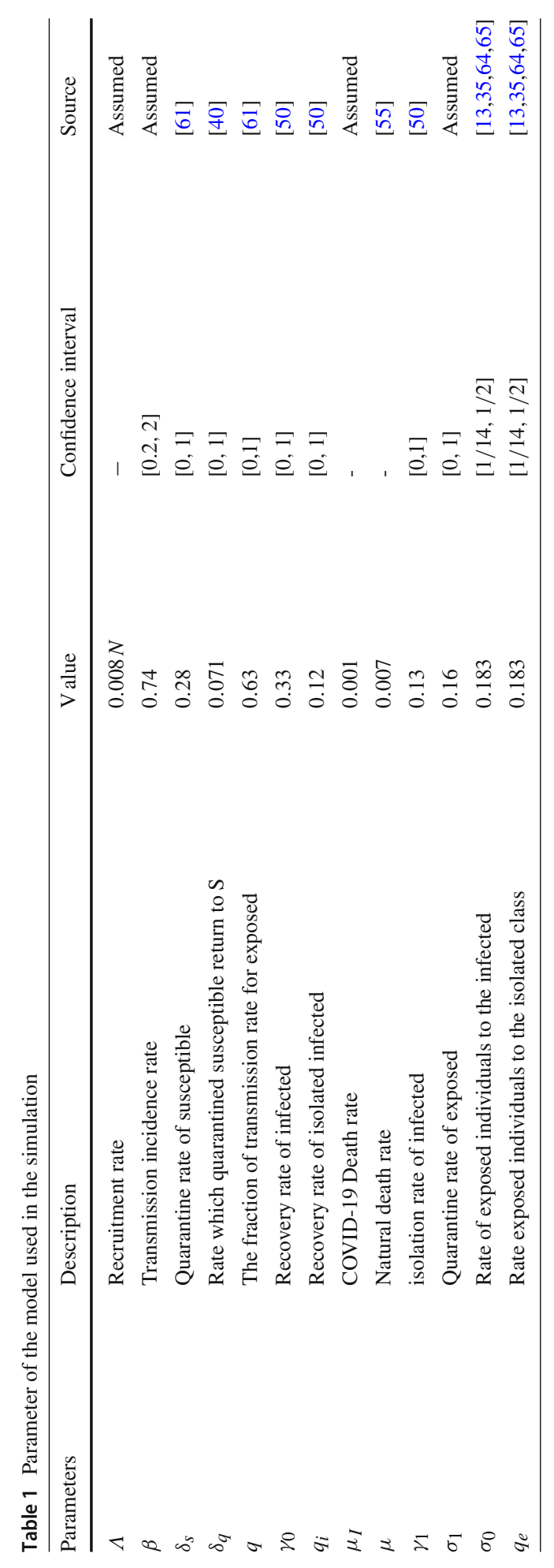




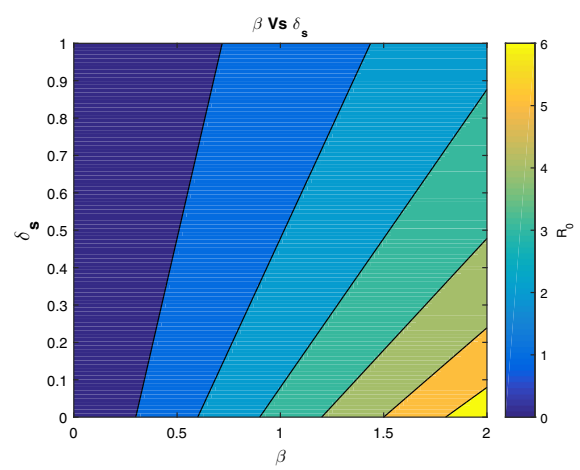

(a) $\beta$ versus $\delta_{s}$

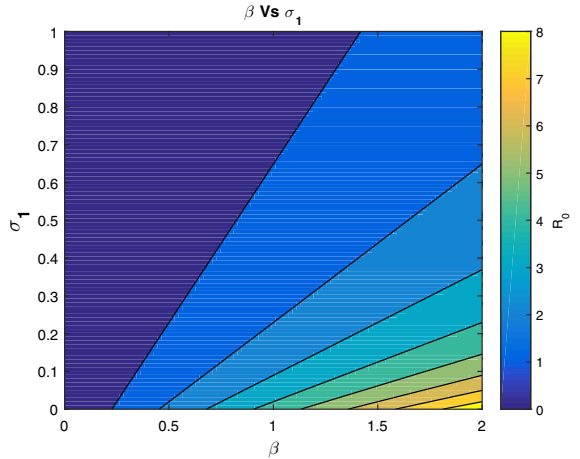

(b) $\beta$ versus $\sigma_{1}$

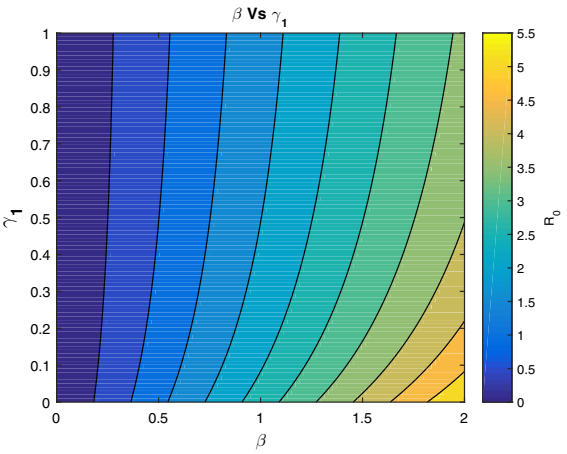

(c) $\beta$ versus $\gamma_{1}$

Fig. 6 Contour shows the variation of $R_{\mathcal{e}}$ for different parameter values: a shows the variation of $R_{0}$ for different values for $\beta$ and $\delta_{s}$, $\mathbf{b}$ shows the variation of $R_{0}$ for different values for $\beta$ and $\sigma_{1}$ and the lower heat map c shows the variation of $R_{0}$ for different values for $\beta$ and $\gamma_{1}$

where

$$
\begin{aligned}
& \tilde{h}_{1}(\alpha)=\frac{1-\alpha}{M(\alpha)}+\frac{55 \alpha h}{24 M(\alpha)} ; \quad \tilde{h}_{2}(\alpha)=-\frac{1-\alpha}{M(\alpha)}-\frac{59 \alpha h}{24 M(\alpha)} ; \\
& \tilde{h}_{3}(\alpha)=\frac{37 \alpha h}{24 M(\alpha)} \text { and } \quad \tilde{h}_{4}(\alpha)=-\frac{9 \alpha h}{24 M(\alpha)} .
\end{aligned}
$$

similarly for the other equations, thus, desired numerical approach is obtained for CFfractional model.

\section{Numerical Simulation and Calibration of the Model}

In this Section, we illustrate the numerical results for the fractional model (2) for different values of fractional order $\alpha$ to analyze the influence of fractional order on the disease dynamics. Some numerical simulations with the Caputo-Fabrizio operator for the fractional COVID-19 virus model are presented using the fractional version of Adams-Bashforth four-step method. 


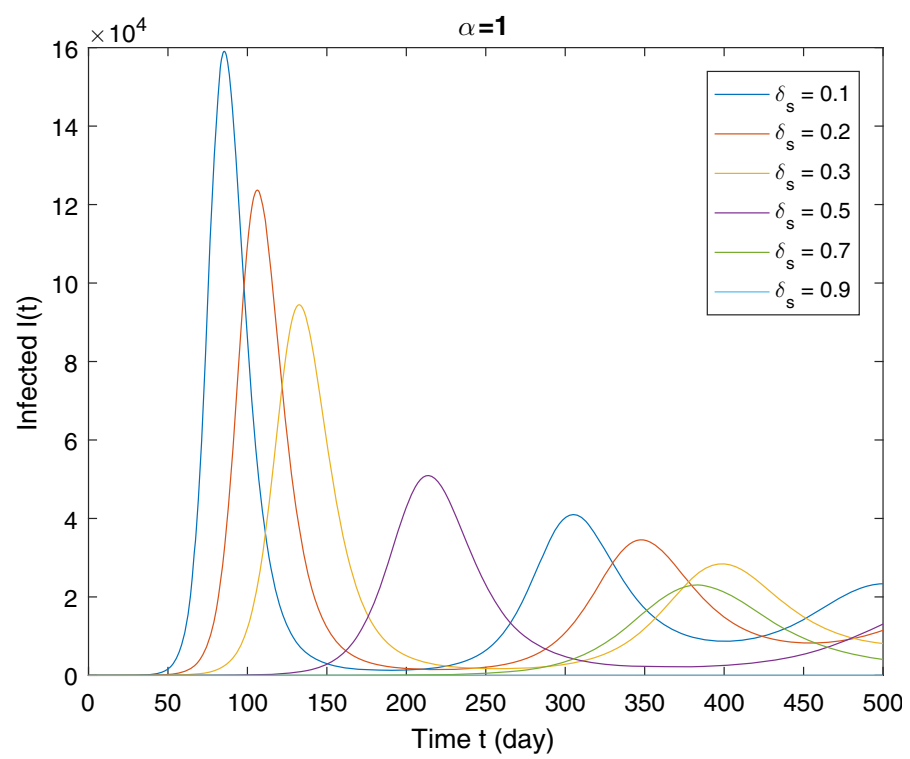

(a) Case $\alpha=1$

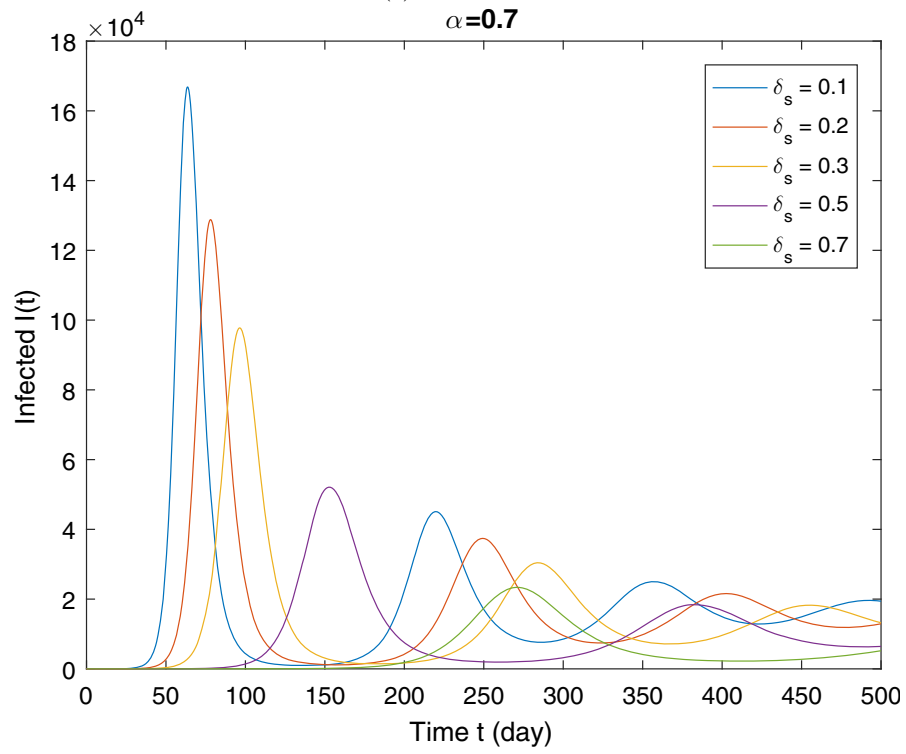

(b) Case $\alpha=0.7$

Fig. 7 Illustration of the impact of quarantine rate $\delta_{S}$ on the dynamics of the infected (I) for two values of $\alpha$. All other parameters are given in the Table 1. The initial conditions are the same of the Section 7.2

\section{Estimation of Model Parameters and Best Fit of Fractional Orders}

The model calibration problem seeks to estimate the model parameters which, to some extent, make the model response as close as possible to the observed values (real data), for that using the Least Squares method to predict the model parameters. 


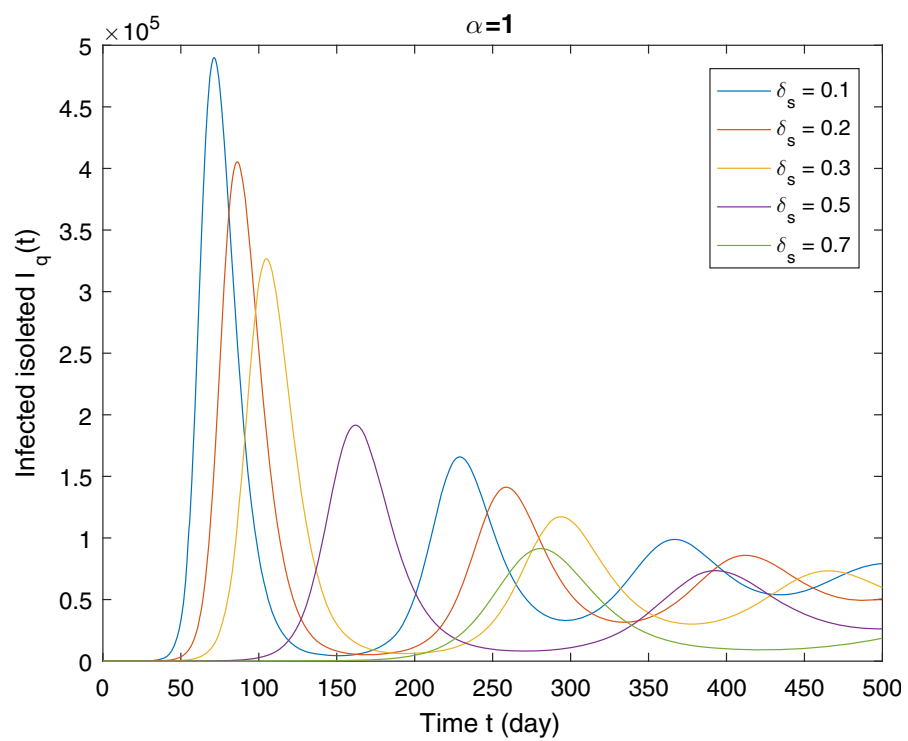

(a) Case $\alpha=1$

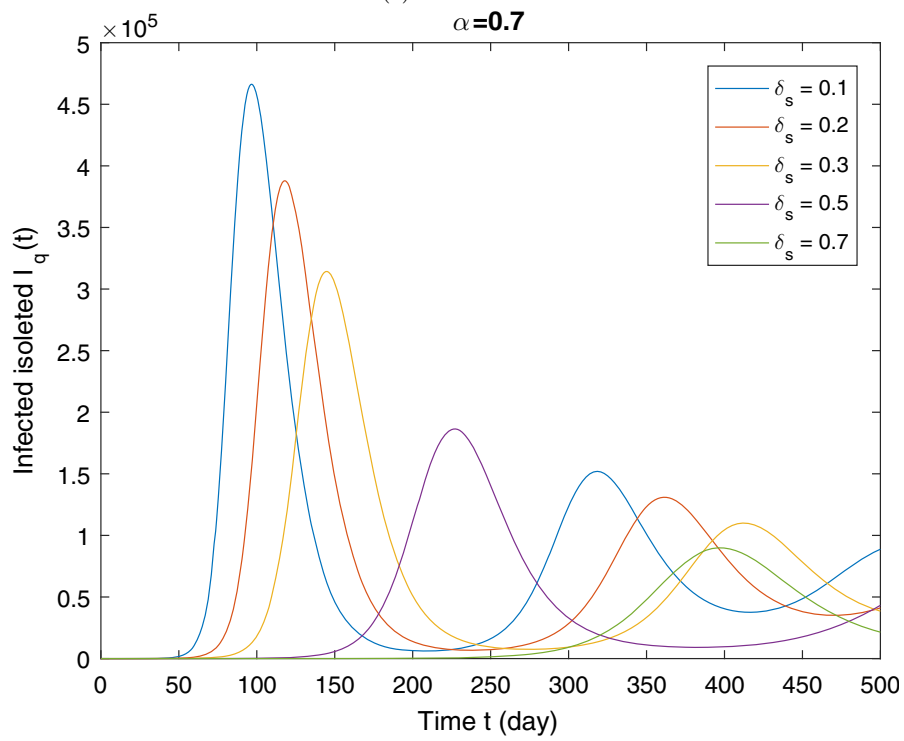

(b) Case $\alpha=0.7$

Fig. 8 Illustration of the impact of quarantine rate $\delta_{s}$ on the dynamics of the infected quarantined $\left(I_{q}\right)$ for two values of $\alpha$. All other parameters are given in the Table 1. The initial conditions are the same of the Section 7.2

We consider the model solution $u(t)=\left(S(t), E(t), I(t), R(t), S_{q}(t), E_{q}(t), I_{q}(t)\right)$, depending on the vector of parameters $\theta=\left(\delta_{s}, \delta_{q}, \beta, q, \sigma_{0}, \gamma_{0}, \sigma_{1}, \gamma_{1}, q_{e}, q_{i}, \mu_{I}\right)$ excepting $\mu, \Lambda$ and $N$, which are kept fixed, and the vector $X$ of the observation data at given times $t_{i}, i=1, \ldots n$. Let $\Psi(u, \theta, \alpha)$ be the function computing the numerical solution $u$ of the 


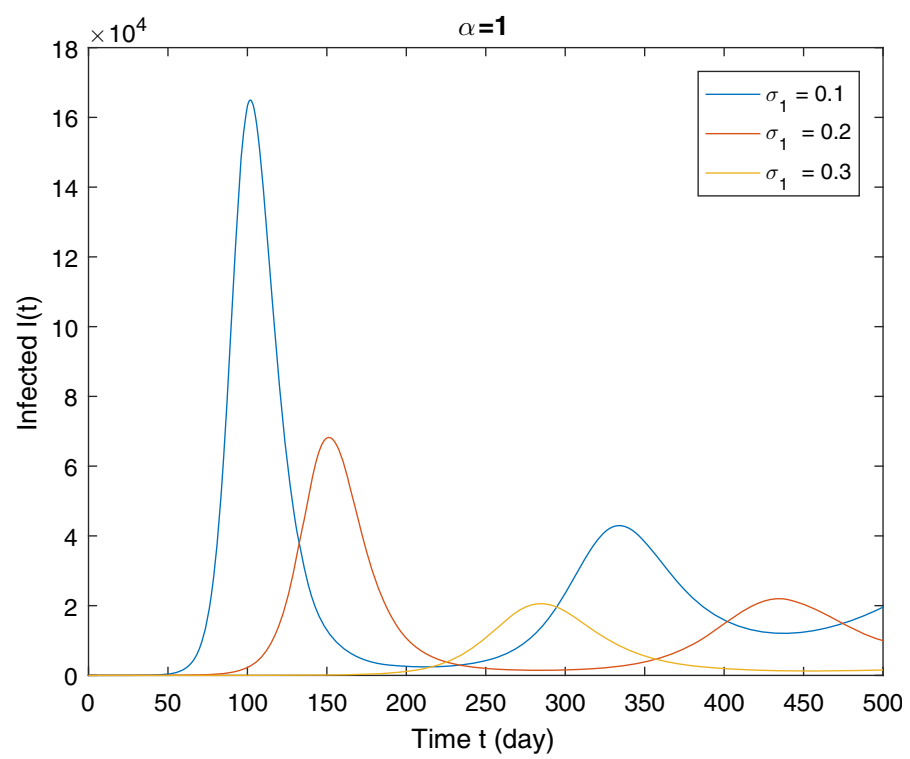

(a) Case $\alpha=1$

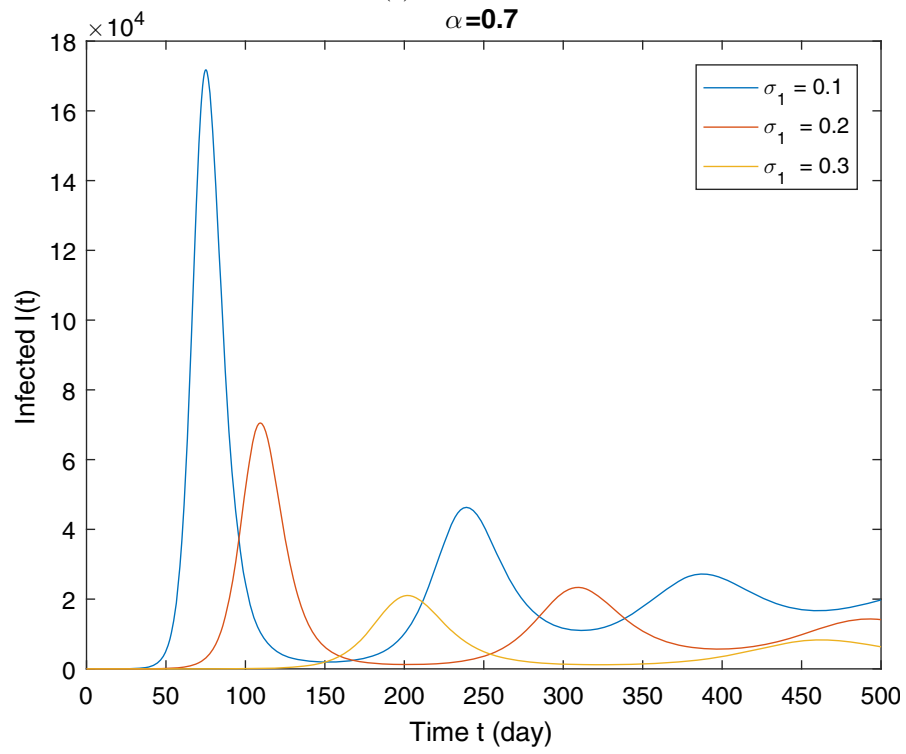

(b) Case $\alpha=0.7$

Fig. 9 Illustration of the impact of quarantine rate $\sigma_{1}$ on the dynamics of the infected (I) for two values of $\alpha$. All other parameters are given in the Table 1. The initial conditions are the same of the Section 7.2

fractional differential system (2), the vector of parameters is restricted to be on the convex set of admissible values $\Theta=\left\{(\theta, \alpha) \in \mathbb{R}^{12} / \mathrm{lb} \leq \theta \leq \mathrm{ub}\right.$ and $\left.0<\alpha \leq 1\right\}$, such as $\mathrm{lb}$ and $\mathrm{ub}$ are lower and upper vector bounds for the model parameter $\theta$ respectively. 


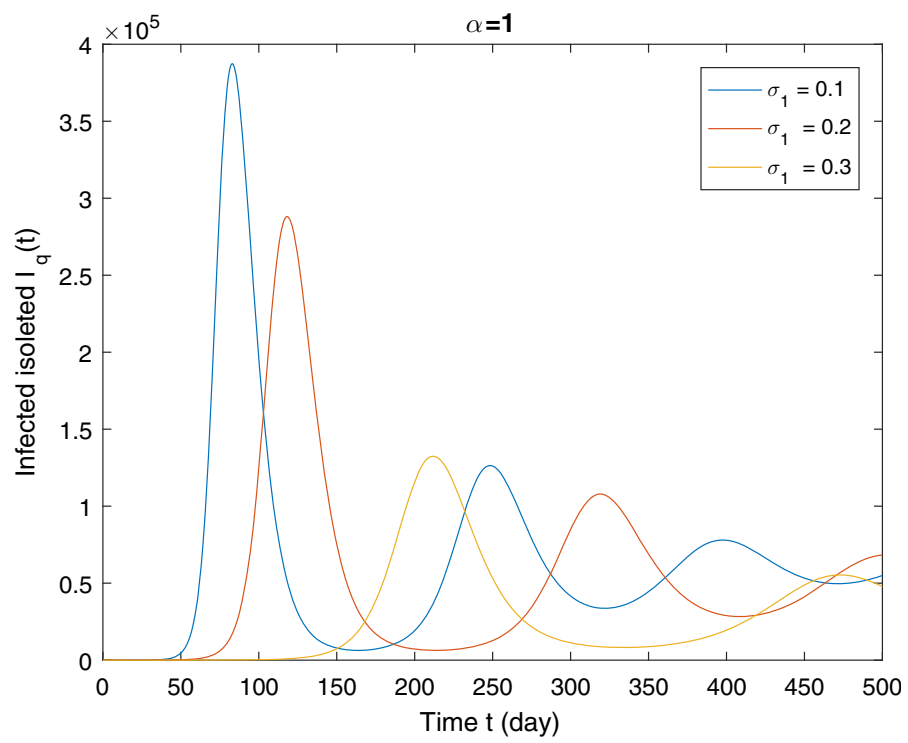

(a) Case $\alpha=1$

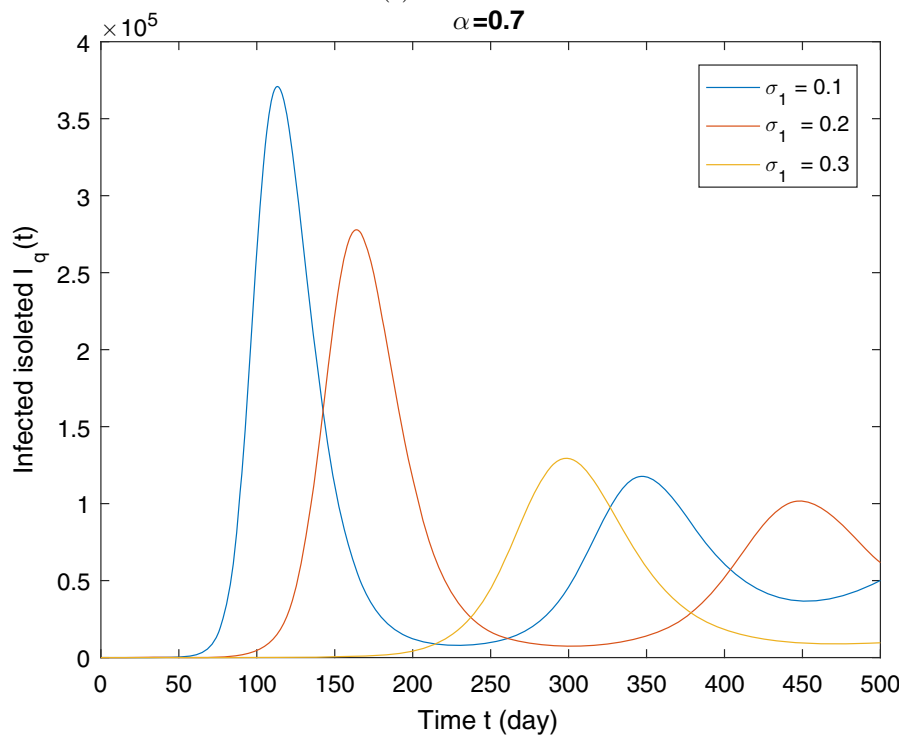

(b) Case $\alpha=0.7$

Fig. 10 Illustration of the impact of quarantine rate $\sigma_{1}$ on the dynamics of the infected quarantined $\left(I_{q}\right)$ for two values of $\alpha$. All other parameters are given in the Table 1. The initial conditions are the same of the Section 7.2

The estimation of the parameter $\theta$ is obtained by solving the following non linear least squares problem by finding a vector of parameter $\theta^{*}$ such that:

$$
\left(\theta^{*}, \alpha^{*}\right)=\underset{(\theta, \alpha) \in \Theta}{\arg \min }\left\{\sum_{j=1}^{n}\left|X_{j}-\Psi\left(u_{j}, \theta, \alpha\right)\right|^{2}\right\} .
$$




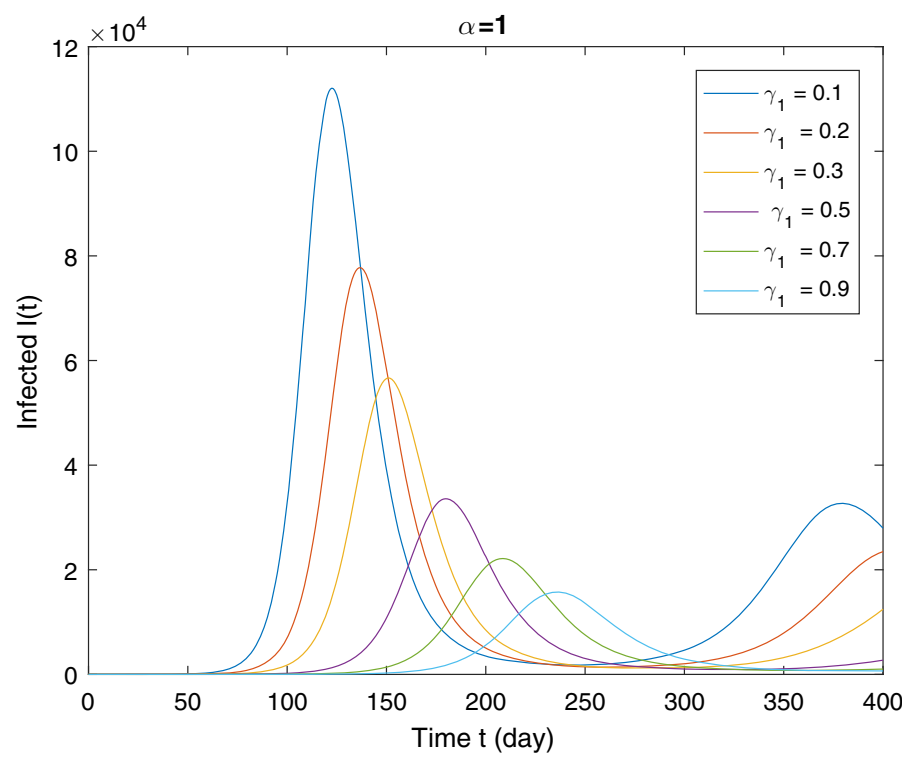

(a) Case $\alpha=1$

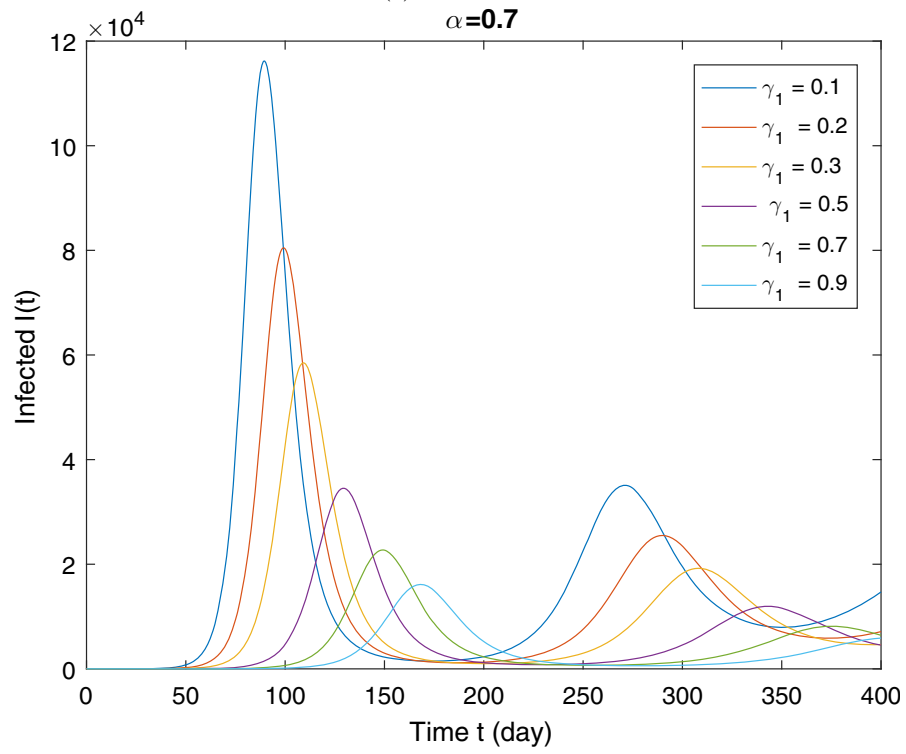

(b) Case $\alpha=0.7$

Fig. 11 Illustration of the impact of isolation rate $\gamma_{1}$ on the dynamics of the infected (I) for two values of $\alpha$. All other parameters are given in the Table 1. The initial conditions are the same of the Section 7.2

The aim is to minimize the objective function

$$
J(\theta, \alpha)=\|X-\Psi(u, \theta, \alpha)\|^{2}=\sum_{j=1}^{n}\left|X_{j}-\Psi\left(u_{j}, \theta, \alpha\right)\right|^{2} .
$$




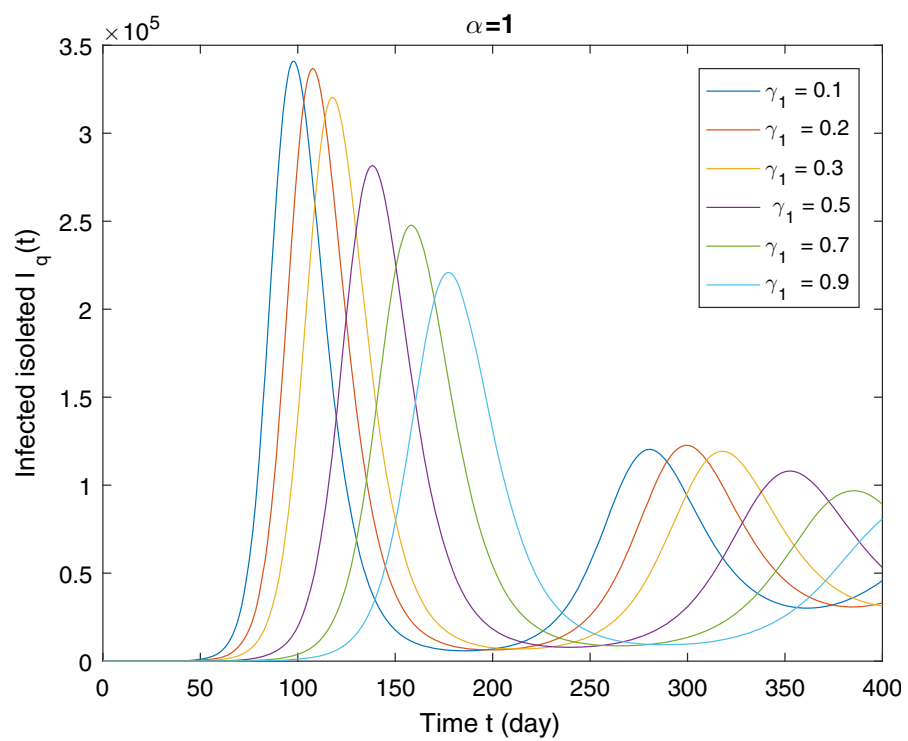

(a) Case $\alpha=1$

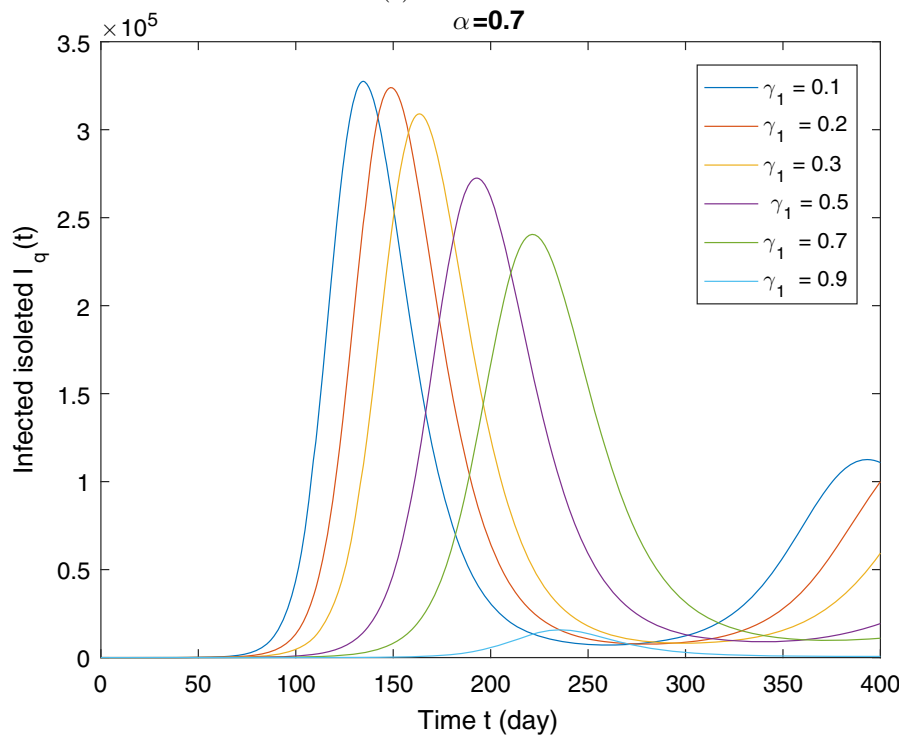

(b) Case $\alpha=0.7$

Fig. 12 Illustration of the impact of isolation rate $\gamma_{1}$ on the dynamics of the infected quarantined $\left(I_{q}\right)$ for two values of $\alpha$. All other parameters are given in the Table 1. The initial conditions are the same of the Section 7.2

subject to $(\theta, \alpha) \in \Theta$ and Equation (2) to obtain the best estimate of parameters and the fractional order $\alpha$. For more details about least squares method, see for example [25,36]. Now using 4-steps Adams-Bashforth method we get the optimization problem 


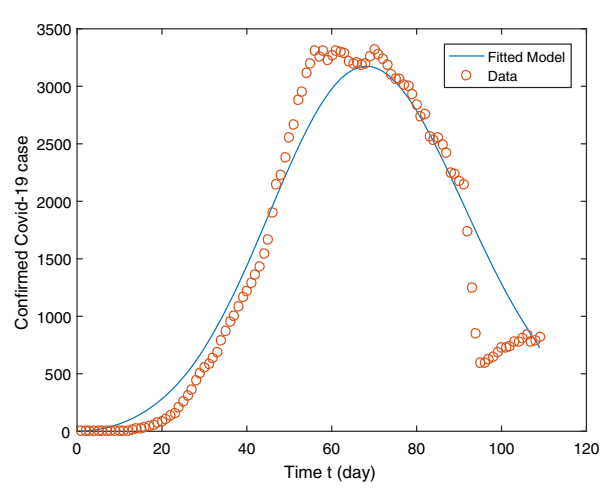

(a) Morocco case from $2^{\text {nd }}$ March to $18^{\text {th }}$ June 2020

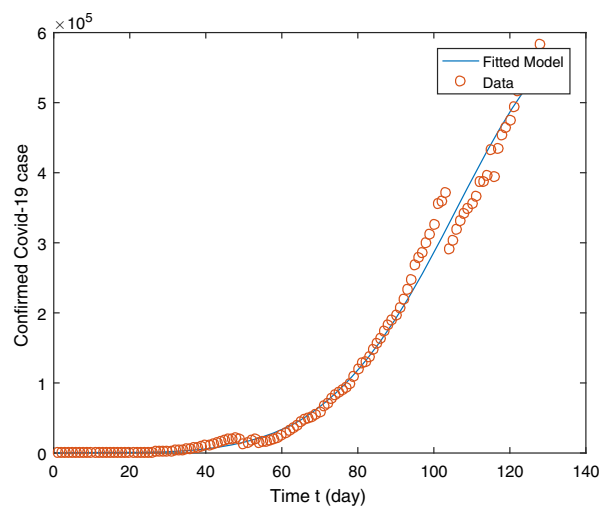

(c) Brazil case from $26^{\text {th }}$ Feburary to $2^{\text {nd }}$ July 2020

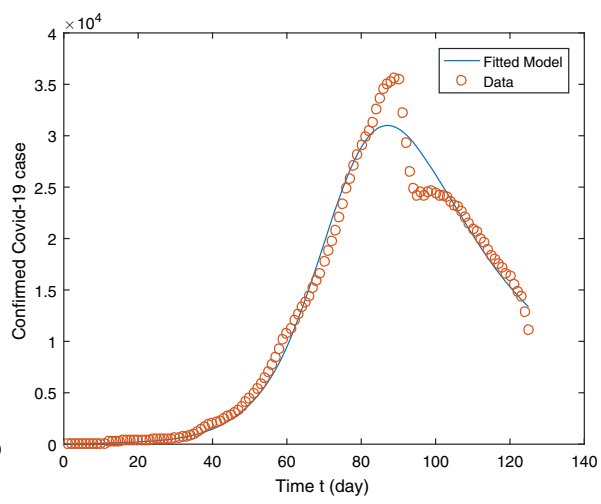

(b) Qatar case from $29^{\text {th }}$ Feburary to $2^{\text {nd }}$ July 2020

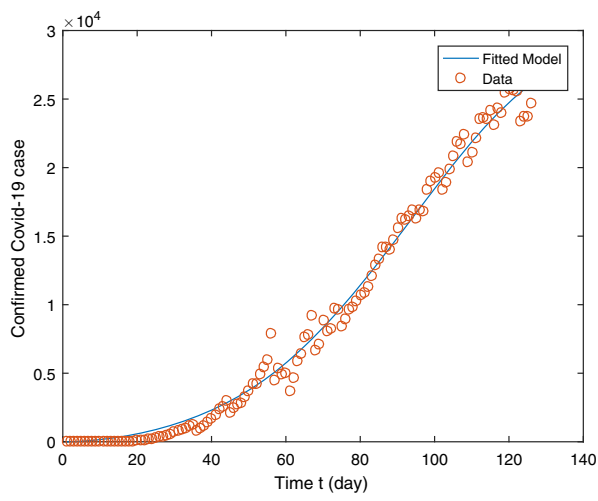

(d) Mexico case from $28^{\text {th }}$ Feburary to $2^{\text {nd }}$ July 2020

Fig. 13 Fitting model to data in Morocco, Qatar, Brazil and Mexico (Real data source: WHO report of COVID-19)

$$
\left\{\begin{array}{l}
\min \left(J(\theta, \alpha)=\sum_{j=1}^{n}\left|X_{j}-\Psi\left(u_{j}, \theta, \alpha\right)\right|^{2}\right), \\
\text { subject to }(\theta, \alpha) \in \Theta, \\
\Psi\left(u_{0}, \theta, \alpha\right)=\left(S_{0}, E_{0}, I_{0}, R_{0}, S_{q, 0}, E_{q, 0}, I_{q, 0}\right), \\
\Psi\left(u_{1}, \theta, \alpha\right)=\Psi\left(u_{0}, \theta, \alpha\right)+\left(K_{1}(\alpha)+K_{2}(\alpha)\right) f\left(t_{0}, \Psi\left(u_{0}, \theta, \alpha\right)\right), \\
\Psi\left(u_{2}, \theta, \alpha\right)=\Psi\left(u_{0}, \theta, \alpha\right)+K_{1}(\alpha) f\left(t_{0}, \Psi\left(u_{0}, \theta, \alpha\right)\right)+K_{2}(\alpha) f\left(t_{1}, \Psi\left(u_{1}, \theta, \alpha\right)\right), \\
\Psi\left(u_{3}, \theta, \alpha\right)=\Psi\left(u_{1}, \theta, \alpha\right)+K_{1}(\alpha) f\left(t_{1}, \Psi\left(u_{1}, \theta, \alpha\right)\right)+K_{2}(\alpha) f\left(t_{2}, \Psi\left(u_{2}, \theta, \alpha\right)\right), \\
\text { for } j=4 \text { until n calculate } \\
\Psi\left(u_{j}, \theta, \alpha\right)=\Psi\left(u_{j-1}, \theta, \alpha\right)+\tilde{h}_{1}(\alpha) f\left(t_{j-1}, \Psi\left(u_{j-1}, \theta, \alpha\right)\right) \\
+\tilde{h}_{2}(\alpha) f\left(t_{j-2}, \Psi\left(u_{j-2}, \theta, \alpha\right)\right)+\tilde{h}_{3}(\alpha) f\left(t_{j-3}, \Psi\left(u_{j-3}, \theta, \alpha\right)\right) \\
+\tilde{h}_{4}(\alpha) f\left(t_{j-4}, \Psi\left(u_{j-4}, \theta, \alpha\right)\right) .
\end{array}\right.
$$

to obtain the best estimate of parameters and the vector of fractional orders $\alpha$, where

$$
K_{1}(\alpha)=\frac{2-2 \alpha+3 \alpha h}{2 M(\alpha)}, \quad K_{2}(\alpha)=\frac{2-2 \alpha+\alpha h}{2 M(\alpha)},
$$


the values of $\tilde{h}_{i}(\alpha), i=1,2,3,4$ are given in (31) and the function $f$ is the right-hand side of the model (2).

\section{Impact of Fractional Order Derivative}

Based on numerical scheme derived in Section 6, we present the graphical results of the proposed CF fractional model (2) in Figs. 2, 3, 4 and 5 to analyze the influence of fractional order. We have considered the initial conditions $N=3500001, S(0)=3500000, I(0)=1$ and $E(0)=R(0)=S_{q}(0)=E_{q}(0)=I_{q}(0)=0$.

From the analysis of the obtained graphs, we notice that the population of the infected class decreases significantly by decreasing the order of the fractional derivative and the endemic state of the disease goes to the disease-free state in all the cases studied. In addition, the graphical results of the CF-derivative model reveal that this operator is more significant in exploring the dynamics of the model and providing more biologically feasible results.

\section{Impact of Quarantined and Isolation Parameters}

It is well known that the effective reproduction number $\left(R_{e}\right)$ of the fractional model is a very important parameter in the infectious disease, which determines whether the could spread. In our model, $R_{e}$ is determined by the parameters given in (3) and (7). In order to identify the impacts of these parameters on COVID-19 transmission and prevalence, we will discuss the change in the number of infected individuals with the COVID-19 virus when we change the values of the controlled parameters, transmission rate $(\beta)$, quarantine rate of susceptible $\left(\delta_{s}\right)$, isolation rate of infected individuals $\left(\gamma_{1}\right)$ and quarantine rate of exposed $\left(\sigma_{1}\right)$. All parameter values for the simulation in this section are given in Table 1.

Firstly, to determine the dependence of the attack rate on the controllable model parameters, fixing two of $\delta_{s}, \sigma_{1}$ and $\gamma_{1}$ at the specific value (given in Table 1) and varying the other two parameters (one of the previous four parameters with $\beta$ ), the contour plots of the quarantine reproduction number are illustrated in Fig. 6.

Figure 6a and $6 \mathrm{~b}$ show that higher quarantine rate $\delta_{s}$ and $\sigma_{1}$ will reduce $R_{0}$, then will help bring $R_{0}<1$, thus prevent the outbreak from happening. On the other hand Fig. 6c shows that the isolation rate is effective when the transmission rate is high, this is natural since infected people are those who carry the virus and transmit it to other people in the population.

In order to compare the effectiveness of quarantine and isolation to reduce the spread of a virus COVID-19, the model is simulated the impact of using quarantine only as well as isolation interventions only for a range of values of quarantine and isolation parameters.

Figures 7, 8, 9 and 10 show the effectiveness of the quarantine strategy, similarly Figs. 11 and 12 show the effectiveness of the isolation of infected cases to control the evolution of viruses.

Table 2 Different values of quarantine reproduction number according to quarantine rate of susceptible individuals

\begin{tabular}{lllllll}
\hline Values of $\delta_{q}$ & 0.1 & 0.2 & 0.3 & 0.5 & 0.7 & 0.9 \\
\hline Values of $R_{e}\left(\sigma_{1}=0.16\right)$ & 2.1652 & 1.9290 & 1.7394 & 1.4535 & 1.2484 & 1.0940 \\
Values of $R_{e}\left(\sigma_{1}=0.36\right)$ & 1.3778 & 1.2276 & 1.1069 & 0.9250 & 0.7944 & 0.6962 \\
\hline
\end{tabular}


Table 3 Different values of quarantine reproduction number according to quarantine rate of exposed individuals

\begin{tabular}{lllllll}
\hline Vlues of $\sigma_{1}$ & 0.1 & 0.2 & 0.3 & 0.5 & 0.7 & 0.9 \\
\hline Values of $R_{e}\left(\delta_{s}=0.28\right)$ & 2.1413 & 1.5923 & 1.2673 & 0.9000 & 0.6977 & 0.5697 \\
\hline
\end{tabular}

Table 4 Initial values using in Fig. 13

\begin{tabular}{lclrrlrll}
\hline Country & $N(0)[57]$ & $S(0)$ & $E(0)$ & $I(0)$ & $R(0)$ & \multicolumn{1}{l}{$S_{q}(0)$} & $E_{q}(0)$ & $I_{q}(0)$ \\
\hline Morocco & 36029093 & $3.3 \times 10^{7}$ & 9 & 1 & 0 & 3029079 & 3 & 1 \\
Qatar & 2881053 & $2 \times 10^{6}$ & 58 & 15 & 0 & 880978 & 1 & 1 \\
Mexico & 128929303 & $1.2 \times 10^{8}$ & 900 & 1 & 0 & 8928398 & 3 & 1 \\
Brazil & 212569392 & $1.6764 \times 10^{7}$ & 227 & 9 & 0 & 195805154 & 1 & 1 \\
\hline
\end{tabular}

\section{Fitting Model to Real Data}

In this present section we have estimated the important model parameters and the fractional order $\alpha$ using the infection cases (that are found on the WHO web-page) in Morocco, Qatar, Brazil and Mexico. The initial conditions used are given in the Table 4, we estimated the value of the fractional order $\alpha$ for the four countries and we found 0.9533 for the Morocco model, 0.9418 for Qatar, 0.9906 for Brazil and 0.822 for Mexico. These values show the importance of the fractional order in the COVID-19 considered model and its influence to decrease the error of the of least squares method. In addition to making the proposed model more realistic and obtaining the average values of the acceptable parameters.

Now by analyzing the curves presented in Fig. 13, the importance role of quarantine and isolation in Morocco and Qatar in reducing the impact of the COVID-19, whilst Mexico and Brazil did not succeed in applying the quarantine well, which caused an increase in the number of people infected with the virus. Finally these values are kept low compared to potential values in the absence of a quarantine and isolation strategy (see Fig. 6, Tables 2 and 3 )

\section{Conclusion}

In this manuscript, a Caputo-Fabrizio fractional differential equation model for COVID-19 with quarantine (of susceptible and exposed cases) and isolation (of symptomatic cases) has been investigate and the effect of each of them was studied. The existence and uniqueness of the system of solutions of the Caputo-Fabrizio model are established using a fixed-point theorem and an Picard iterative method. The m-step Adams-Bashforth approach is proposed to numerically approximate the solutions of the fractional model. We have compared the numerical simulations with respect to different values of the fractional order $\alpha$. Finally, The Method of Least Squares is used to determine the best fit to real data (real data for Morocco, Qatar, Brazil and Mexico). The paper gives an example of the use of the CaputoFabrizio fractional derivative as a model for real world problems (especially epidemiological problems). 
Acknowledgements The authors would like to thank the anonymous reviewers for their insightful suggestions and careful reading of the manuscript.

\section{References}

1. Abdo, M.S., Shah, K., Wahash, H.A., Panchal, S.K.: On a comprehensive model of the novel coronavirus (COVID-19) under Mittag-Leffler derivative. Chaos Solitons Fractals 135, 109867 (2020). https://doi. org/10.1016/j.chaos.2020.109867. ISSN 0960-0779

2. Ahmad, Z., Arif, M., Ali, F., Khan, I., Nisar, K.S.: A report on covid-19 epidemic in pakistan using seir fractional model. Sci. Rep. 10(1), 1-14 (2020)

3. Alaoui, A.L., Azroul, E., Hamou, A.A.: Monotone iterative technique for nonlinear periodic time fractional parabolic problems. Adv. Theory Nonlinear Anal. Appl. 4(3), 194-213 (2020)

4. Alkahtani, B.S.T., Alzaid, S.S.: A novel mathematics model of covid-19 with fractional derivative stability and numerical analysis. Chaos, Solitons Fractals 138, 110006 (2020). https://doi.org/10.1016/j.chaos. 2020.110006. ISSN 0960-0779

5. Alshomrani, A.S., Ullah, M.Z., Baleanu, Dumitru: Caputo sir model for covid-19 under optimized fractional order. Adv. Differ. Equ. 2021(1), 1-17 (2021)

6. Area, I., Batarfi, H., Losada, J., Nieto, J.J., Shammakh, W., Torres, Á.: On a fractional order Ebola epidemic model. Adv. Differ. Equ. 2015(1), 278 (2015). https://doi.org/10.1186/s13662-015-0613-5

7. Atangana, A.: Modelling the spread of COVID-19 with new fractal-fractional operators: Can the lockdown save mankind before vaccination? Chaos Solitons Fractals 136, 109860 (2020). https://doi.org/10.1016/ j.chaos.2020.109860. ISSN 0960-0779

8. Atangana, A., Baleanu, D.: New fractional derivatives with nonlocal and non-singular kernel: theory and application to heat transfer model. Therm. Sci. (2016). https://doi.org/10.2298/TSCI160111018A

9. Atangana, A., Baleanu, D.: Caputo-fabrizio derivative applied to groundwater flow within confined aquifer. J. Eng. Mech. 143(5), D4016005 (2017). https://doi.org/10.1061/(ASCE)EM.1943-7889. 0001091

10. Atangana, A., Koca, I.: Chaos in a simple nonlinear system with atangana-baleanu derivatives with fractional order. Chaos Solitons Fractals 89, 447-454 (2016). https://doi.org/10.1016/j.chaos.2016.02. 012. ISSN 0960-0779

11. Atangana, A., Owolabi, K.M.: New numerical approach for fractional differential equations. Math. Model. Nat. Phenom. 13(1), 3 (2018). https://doi.org/10.1051/mmnp/2018010

12. Baba, I.A., Yusuf, A., Nisar, K.S., Abdel-Aty, A.H., Nofal, T.A.: Mathematical model to assess the imposition of lockdown during covid-19 pandemic. Results Phys. 20, 103716 (2021)

13. Backer, J.A., Klinkenberg, D., Wallinga, J.: Incubation period of 2019 novel coronavirus (2019-nCoV) infections among travellers from Wuhan, China, 20-28 january 2020. Eurosurveillance 25(5), 2000062 (2020). https://doi.org/10.2807/1560-7917.ES.2020.25.5.2000062

14. Baleanu, D., Aydogn, S.M., Mohammadi, H., Rezapour, S.: On modelling of epidemic childhood diseases with the Caputo-Fabrizio derivative by using the Laplace Adomian decomposition method. Alex. Eng. J. 59(5), 3029-3039 (2020)

15. Baleanu, D., Etemad, S., Rezapour, S.: A hybrid caputo fractional modeling for thermostat with hybrid boundary value conditions. Bound. Value Probl. 2020(1), 1-16 (2020)

16. Baleanu, D., Jajarmi, A., Mohammadi, H., Rezapour, S.: A new study on the mathematical modelling of human liver with caputo-fabrizio fractional derivative. Chaos Solitons Fractals 134, 109705 (2020)

17. Baleanu, D., Mohammadi, H., Rezapour, S.: Analysis of the model of HIV-1 infection of CD4 ${ }^{+}$T-cell $^{-}$ with a new approach of fractional derivative. Adv. Differ. Equ. 2020(1), 1-17 (2020)

18. Boudaoui, A., ElhadjMoussa, Y., Hammouch, Z., Ullah, S.: A fractional-order model describing the dynamics of the novel coronavirus (covid-19) with nonsingular kernel. Chaos Solitons Fractals 146, 110859 (2021)

19. Caputo, M., Fabrizio, M.D.: A new definition of fractional derivative without singular kernel. Progr. Fract. Differ. Appl. 1(2), 1-13 (2015)

20. Castillo-Chavez, C., Castillo-Garsow, C.W., Yakubu, A.: Mathematical models of isolation and quarantine. JAMA 290(21), 2876-2877 (2003). https://doi.org/10.1001/jama.290.21.2876

21. Cheema, S.A., Kifayat, T., Rahman, A.R., Khan, U., Zaib, A., Khan, I., Nisar, K.S.: Is social distancing, and quarantine effective in restricting covid-19 outbreak? statistical evidences from Wuhan, China. Comput. Mater. Contin. 66(2) (2020) 
22. Chimmalee, B., Sawangtong, W., Wiwatanapataphee, B.: The effects of community interactions and quarantine on a complex network. Cogent Math. 3(1), 1249141 (2016). https://doi.org/10.1080/23311835. 2016.1249141

23. Cohen, J.: New SARS-like virus in China triggers alarm. Science 367(6475), 234-235 (2020). https:// doi.org/10.1126/science.367.6475.234. ISSN 0036-8075

24. Danane, J., Allali, K., Hammouch, Z., Nisar, K.S.: Mathematical analysis and simulation of a stochastic covid-19 lévy jump model with isolation strategy. Results Phys. 23, 103994 (2021)

25. Dantas, E., Tosin, M., Cunha Jr., A.: Calibration of a SEIR-SEI epidemic model to describe the Zika virus outbreak in brazil. Appl. Math. Comput. 338, 249-259 (2018). https://doi.org/10.1016/j.amc.2018. 06.024

26. Dokuyucu, M.A., Dutta, H.: A fractional order model for Ebola virus with the new Caputo fractional derivative without singular kernel. Chaos Solitons Fractals 134, 109717 (2020). https://doi.org/10.1016/ j.chaos.2020.109717. ISSN 0960-0779

27. Erdem, M., Safan, M., Castillo-Chavez, C.: Mathematical analysis of an SIQR influenza model with imperfect quarantine. Bull. Math. Biol. 79(7), 1612-1636 (2017). https://doi.org/10.1007/s11538-0170301-6

28. Ghanbari, B., Atangana, A.: A new application of fractional atangana-baleanu derivatives: Designing abc-fractional masks in image processing. Phys. A 542, 123516 (2020). https://doi.org/10.1016/j.physa. 2019.123516. ISSN 0378-4371

29. Hamou, A.A., Azroul, E.H., Hammouch, Z., Alaoui, A.L.: A fractional multi-order model to predict the COVID-19 outbreak in Morocco. Appl. Comput. Math. 20(1), 177-203 (2020)

30. Higazy, M.: Novel fractional order SIDARTHE mathematical model of COVID-19 pandemic. Chaos Solitons Fractals 138, 110007 (2020). https://doi.org/10.1016/j.chaos.2020.110007. ISSN 0960-0779

31. Hussain, G., Khan, T., Khan, A., Inc, M., Zaman, G., Nisar, K.S., Akgül, A.: Modeling the dynamics of novel coronavirus (covid-19) via stochastic epidemic model. Alex. Eng. J. 60(4), 4121-4130 (2021)

32. Jorge, L., Nieto, J.J.: Properties of a new fractional derivative with out singular kernel. Prog. Fract. Differ. Appl. Int. J. 87-92 (2015). http://www.naturalspublishing.com/files/published/2j1ns3h8o2s789.pdf

33. Kumar, D., Singh, J., Al Qurashi, M., Baleanu, D.: A new fractional SIRS-SI malaria disease model with application of vaccines, antimalarial drugs, and spraying. Adv. Differ. Equ. 2019(1), 278 (2019). https:// doi.org/10.1186/s13662-019-2199-9

34. Kumar, S., Cao, J., Abdel-Aty, M.: A novel mathematical approach of COVID-19 with non-singular fractional derivative. Chaos Solitons Fractals 139, 110048 (2020). https://doi.org/10.1016/j.chaos.2020. 110048. ISSN 0960-0779

35. Lauer, Stephen A., Grantz, Kyra H., Bi, Qifang, Jones, Forrest K., Zheng, Qulu, Meredith, Hannah R., Azman, Andrew S., Reich, Nicholas G., Lessler, Justin: The incubation period of coronavirus disease 2019 (COVID-19) from publicly reported confirmed cases: estimation and application. Ann. Intern. Med. 172(9), 577-582 (2020). https://doi.org/10.7326/M20-0504

36. Li, M.Y.: An Introduction to Mathematical Modeling of Infectious Diseases. Springer, New York (2018)

37. Logeswari, K., Ravichandran, C., Nisar, K.S.: Mathematical model for spreading of covid-19 virus with the mittag-leffler kernel. Numer. Methods Partial Differ. Equ. (2020)

38. Mohammadi, H., Kumar, S., Rezapour, S., Etemad, S.: A theoretical study of the caputo-fabrizio fractional modeling for hearing loss due to mumps virus with optimal control. Chaos Solitons Fractals 144, 110668 (2021)

39. Moore, E.J., Sirisubtawee, S., Koonprasert, S.: A caputo-fabrizio fractional differential equation model for HIV/AIDS with treatment compartment. Adv. Diff. Equ. 2019(1), 200 (2019). https://doi.org/10.1186/ s13662-019-2138-9

40. National Health Commission of the People's Republic of China: Accessed: (23 January 2020). http:// www.nhc.gov.cn/xcs/xxgzbd/gzbd_index.shtml

41. Nisar, K.S., Ahmad, S., Ullah, A., Shah, K., Alrabaiah, H., Arfan, M.: Mathematical analysis of sird model of covid-19 with caputo fractional derivative based on real data. Results Phys. 21, 103772 (2021)

42. Peter, O.J., Shaikh, A.S., Ibrahim, M.O., Nisar, K.S., Baleanu, D., Khan, I., Abioye A.I.: Analysis and dynamics of fractional order mathematical model of covid-19 in nigeria using atangana-baleanu operator. Comput. Mater. Contin. 66(2), (2020)

43. Rezapour, S., Etemad, S., Mohammadi, H.: A mathematical analysis of a system of Caputo-Fabrizio fractional differential equations for the anthrax disease model in animals. Adv. Differ. Equ. 2020(1), 1-30 (2020)

44. Rezapour, S., Mohammadi, H., Jajarmi, A.: A new mathematical model for Zika virus transmission. Adv. Differ. Equ. 2020(1), 1-15 (2020)

45. Sahoo, P., Mondal, H.S., Hammouch, Z., Abdeljawad, T., Mishra, D., Reza, M.: On the necessity of proper quarantine without lock down for 2019-ncov in the absence of vaccine. Results Phys. 25, 104063 (2021) 
46. Shaikh, A.S., Shaikh, I.N., Nisar, K.S.: A mathematical model of covid-19 using fractional derivative: outbreak in india with dynamics of transmission and control. Adv. Differ. Equ. 2020(1), 1-19 (2020)

47. Singh, J., Kumar, D., Baleanu, D.: On the analysis of fractional diabetes model with exponential law. Adv. Differ. Equ. 2018(1), 1-15 (2018). https://doi.org/10.1186/s13662-018-1680-1

48. Singh, J., Kumar, D., Hammouch, Z., Atangana, A.: A fractional epidemiological model for computer viruses pertaining to a new fractional derivative. Appl. Math. Comput. 316, 504-515 (2018). https://doi. org/10.1016/j.amc.2017.08.048

49. Singh, H., Srivastava, H.M., Hammouch, Z., Nisar, K.S.: Numerical simulation and stability analysis for the fractional-order dynamics of covid-19. Results Phys. 20, 103722 (2021)

50. Tang, B., Wang, X., Li, Q., Bragazzi, N.L., Tang, S., Xiao, Y., Jianhong, W.: Estimation of the transmission Risk of the 2019-nCoV and its implication for public health interventions. J. Clin. Med. 9(2), 462 (2020). https://doi.org/10.3390/jcm9020462

51. Thabet, S.T.M., Etemad, S., Rezapour, S.: On a new structure of the pantograph inclusion problem in the Caputo conformable setting. Bound. Value Probl. 2020(1), 1-21 (2020)

52. Toufik, M., Atangana, A.: New numerical approximation of fractional derivative with non-local and nonsingular kernel: application to chaotic models. Eur. Phys. J. Plus 132(10), 444 (2017). https://doi.org/10. 1140/epjp/i2017-11717-0

53. Tuan, N.H., Mohammadi, H., Rezapour, S.: A mathematical model for covid-19 transmission by using the caputo fractional derivative. Chaos Solitons Fractals 140, 110107 (2020)

54. Tulu, T.W., Tian, B., Zunyou, W.: Modeling the effect of quarantine and vaccination on Ebola disease. Adv. Differ. Equ. 2017(1), 1-14 (2017). https://doi.org/10.1186/s13662-017-1225-z

55. United Nations Population Division: UNdata: Crude death rate per 1000 population, Accessed: (17 June 2020). http://data.un.org/Data.aspx?q=world+death+rate\&d=PopDiv\&f=variableID\%3a65 $\% 3$ bcrID $\% 3$ a $1802 \% 2 \mathrm{c} 900$

56. Van den Driessche, P., Watmough, J., Pauline, W.: Reproduction numbers and sub-threshold endemic equilibria for compartmental models of disease transmission. Math. Biosci. 180(1-2), 29-48 (2002). https://doi.org/10.1016/S0025-5564(02)00108-6

57. Worldometer: International team of developers, researchers and volunteers, Accessed: (5 July 2020). https://www.worldometers.info/world-population

58. Yang, X.J., Baleanu, D.: Fractal heat conduction problem solved by local fractional variation iteration method. Therm. Sci. 17(2), 625-628 (2013). https://doi.org/10.2298/TSCI121124216Y

59. Yang, Q., Chen, D., Zhao, T., Chen, Y.Q.: Fractional calculus in image processing: a review. Fract. Calc. Appl. Anal. 19(5), 1222-1249 (2016). https://doi.org/10.1515/fca-2016-0063

60. Zamir, M., Nadeem, F., Abdeljawad, T., Hammouch, Z.: Threshold condition and non pharmaceutical interventions's control strategies for elimination of covid-19. Results Phys. 20, 103698 (2021)

61. Zengyun, H., Cui, Q., Han, J., Wang, X., Wei, E.I., Teng, Z.: Evaluation and prediction of the COVID-19 variations at different input population and quarantine strategies, a case study in guangdong province, china. Int. J. Infectious Dis. 95, 231-240 (2020). https://doi.org/10.1016/j.ijid.2020.04.010

62. Zhang, Z.: A novel covid-19 mathematical model with fractional derivatives: Singular and nonsingular kernels. Chaos Solitons Fractals (2020). https://doi.org/10.1016/j.chaos.2020.110060. ISSN 0960-0779

63. Zhang, Y., Xiangnan, Y., Sun, H.G., Tick, G.R., Wei, W., Jin, B.: Applicability of time fractional derivative models for simulating the dynamics and mitigation scenarios of COVID-19. Chaos Solitons Fractals 138, 109959 (2020). https://doi.org/10.1016/j.chaos.2020.109959. ISSN 0960-0779

64. Zhao, S., Lin, Q., Ran, J., Musa, S.S., Yang, G., Wang, W., Lou, Y., Gao, D., Yang, L., He, D., Wang, M.H.: Preliminary estimation of the basic reproduction number of novel coronavirus (2019-nCoV) in China, from 2019 to 2020: a data-driven analysis in the early phase of the outbreak. Int. J. Infectious Dis. 92, 214-217 (2020). https://doi.org/10.1016/j.ijid.2020.01.050. ISSN 1201-9712

65. Zhao, S., Musa, S.S., Lin, Q., Ran, J., Yang, G., Wang, W., Lou, Y., Yang, L., Gao, D., He, D., et al.: Estimating the unreported number of novel coronavirus (2019-nCoV) cases in China in the first half of january 2020: A data-driven modelling analysis of the early outbreak. J. Clin. Med. 9(2), 388 (2020). https://doi.org/10.3390/jcm9020388

Publisher's Note Springer Nature remains neutral with regard to jurisdictional claims in published maps and institutional affiliations. 